\title{
M-Type Barium Hexagonal Ferrite Films
}

\author{
Mingzhong $\mathrm{Wu}$ \\ Department of Physics, Colorado State University, Fort Collins,
}

USA

\section{Introduction}

Magnetic garnet materials such as yttrium iron garnet (YIG) have been widely used as active components in many microwave devices.1,2,3 These devices include resonators, filters, circulators, isolators, and phase shifters. They have had a major impact on the advancement of microwave technology. The underlying physical effects in microwave magnetic devices include ferromagnetic resonance (FMR), magnetostatic wave (MSW) propagation, Faraday rotation, and field displacement. Whatever the basis for a given device, the operation frequency is determined essentially by the FMR frequency of the garnet material. The magnetic garnets are low-magnetization, low-magnetocrystalline-anisotropy materials and, therefore, typically have a low FMR frequency in the GHz range. This imposes an upper limit on the practical operation frequency of compact YIG-based devices in the $10-18 \mathrm{GHz}$ frequency range.

Presently, there is a critical need for millimeter ( $\mathrm{mm}$ ) wave devices which operate in the frequency range from about $30 \mathrm{GHz}$ to $100 \mathrm{GHz}, 4,5,6$ This need is critical for three reasons. (1) Millimeter waves are recognized as a broadband frequency resource that can offer various wireless access applications. (2) The need for broadband telecommunication capabilities will mandate the use of $\mathrm{mm}$-wave frequencies in next-generation satellite systems. (3) Electromagnetic radiation at mm-wave frequencies can penetrate clouds, fog, and many kinds of smoke, all of which are generally opaque to visible or infrared light.

In principle, one can extend the operation frequency of current microwave magnetic devices to the mm-wave frequency range through the use of high external magnetic bias fields. In practical terms, however, the use of high external fields is usually impractical because of the increased device size and weight, as well as incompatibility with monolithic integrated circuit technology.

One important strategy for the above-described frequency extension is to use M-type barium hexagonal ferrite $\mathrm{BaFe}_{12} \mathrm{O}_{19}(\mathrm{BaM})$ films as a replacement for those magnetic garnets. $\mathrm{BaM}$ films can have a very high magnetocrystalline anisotropy field. This high internal field can facilitate ferromagnetic resonance and hence device operation at mm-wave frequencies. The films can also have high remanent magnetization that can allow for device operation in absence of external magnetic fields, namely, self-biased operation, and frequency tuning using very low external fields.

To this end, significant efforts have been made in recent years that range from material preparations to structure and property characterizations and also to device applications. 
Emphasis has been placed on the optimization of deposition processes for low-loss, self-biased BaM thin films, $7,8,9,10$ the deposition of BaM thin films on "non-conventional" substrates, such as semiconductor substrates $11,12,13,14$ and metallic substrates, 15 the fabrication of BaM thick films on semiconductor substrates, 16,17 the demonstration of BaM-based planar mm-wave devices, 18,19,20,21,22,23,10,24 the development of BaM-based ferromagnetic/ferroelectric heterostructures, 25,26,27,28,29 and the study of multiferroic effects in single-phase BaM materials. ${ }^{30} \mathrm{~A}$ variety of different techniques have been used to fabricate BaM film materials. These include pulsed laser deposition (PLD), 7,8,9,10,11,12,28,31 liquid phase epitaxy (LPE), 32,33,34,35 RF magnetron sputtering, 36,37,19,38,39,40 molecular beam epitaxy (MBE), 14 metallo-organic decomposition (MOD), 15 chemical vapor deposition (CVD), ${ }^{41}$ and screen printing. ${ }^{16,17}$ The device demonstration includes both numerical 20,21,22 and experimental efforts. 18,21,22,23,10,24 The devices demonstrated include phase shifters, ${ }^{21}$ filters, 22,23,10,24 circulators, 18 and isolators. ${ }^{19}$

This chapter reviews the main advances made in the field of BaM materials and devices over the past five years. Section 2 gives a brief introduction to hexagonal ferrites first and then describes in detail the structure and properties of BaM materials. This section serves to provide a background for the discussions in the following sections. Section 3 reviews the advances made in the development of BaM film materials. Section 3.1 describes the deposition of low-loss, high-remanent-magnetization BaM thin films on sapphire substrates by PLD techniques. ${ }^{10}$ Section 3.2 discusses the deposition of $\mathrm{BaM}$ thin films on metallic substrates by the MOD method. ${ }^{15}$ Section 3.3 reviews the deposition of BaM thin films on semiconductor substrates by PLD and MBE techniques.13,14 Section 3.4 describes the fabrication of BaM thick films on semiconductor substrates by screen printing. ${ }^{16,17}$ Section 4 reviews the demonstration of $\mathrm{BaM}$ thin film-based mm-wave notch filters ${ }^{10,24}$ and phase shifters. ${ }^{21}$ Finally, Section 5 discusses future work in the field of BaM materials and devices.

\section{Structure and properties of M-type barium hexagonal ferrites (BaM)}

\subsection{Building blocks of hexagonal ferrites}

In many solids, the atoms look like attracting hard spheres and are packed as closely as possible. ${ }^{42,43}$ Figure 1 shows a close-packed layer of identical spheres which occupy positions A. This layer is formed by placing each sphere in contact with six others in a plane. A second and identical layer of spheres can be placed on top of this layer and occupy positions B. Each sphere in the second layer is in contact with three spheres in the first layer. A third layer of spheres may be added in two ways: they can occupy either positions A or positions C. In principle, there are an infinite number of ways of stacking the close-packed layers. Two very common stacking sequences are "ABAB..." and "ABCABC...". The first one gives a hexagonal close-packed (hcp) structure. The second one gives a structure known as facecentred cubic (fcc).

Hexagonal ferrites consist of close-packed layers of oxygen ions O2- 44,45 In certain layers, some oxygen ions are replaced by barium ions $\mathrm{Ba}^{2+}$, which are approximately of the same size as oxygen ions. These close-packed layers form six fundamental blocks, $S, S^{*}, R_{,} R^{*}, T$, and $T^{*}$, among which the $S^{*}, R^{*}$, and $T^{*}$ blocks can be obtained simply through the rotation of the $S, R$, and T blocks, respectively, by $180^{\circ}$ about the $c$ axis. The different stacking of the fundamental blocks builds up materials with different structures and physical properties. Table I lists the chemical compositions and building blocks of five types of hexagonal ferrites. As indicated in Table I, M-type hexagonal ferrites are built from the stacking of S, R, 
$\mathrm{S}^{*}$, and $\mathrm{R}^{*}$ blocks. The structures of $\mathrm{S}$ and $\mathrm{R}$ blocks are described below. One can refer to Refs. [44] and [45] for the structure of T blocks.

\begin{tabular}{c|c|c}
\hline Type & Formula & Build-up \\
\hline $\mathrm{M}$ & $\mathrm{BaFe}_{12} \mathrm{O}_{19}$ & SRS*R \\
\hline $\mathrm{W}$ & $\mathrm{BaMe}_{2} \mathrm{Fe}_{16} \mathrm{O}_{27}$ & SSRS*S*R* \\
\hline $\mathrm{Y}$ & $\mathrm{Ba}_{2} \mathrm{Me}_{2} \mathrm{Fe}_{12} \mathrm{O}_{22}$ & STST \\
\hline $\mathrm{Z}$ & $\mathrm{Ba}_{3} \mathrm{Me}_{2} \mathrm{Fe}_{24} \mathrm{O}_{41}$ & RSTSR $^{*} \mathrm{~S}^{*} \mathrm{~T}^{*}$ \\
\hline $\mathrm{U}$ & $\mathrm{Ba}_{4} \mathrm{Me}_{2} \mathrm{Fe}_{36} \mathrm{O}_{60}$ & RSR $^{*} \mathrm{~S}^{*} \mathrm{TS}^{*}$ \\
\hline
\end{tabular}

Table I. Compositions and building blocks of five types of hexagonal ferrites $44,45,46$

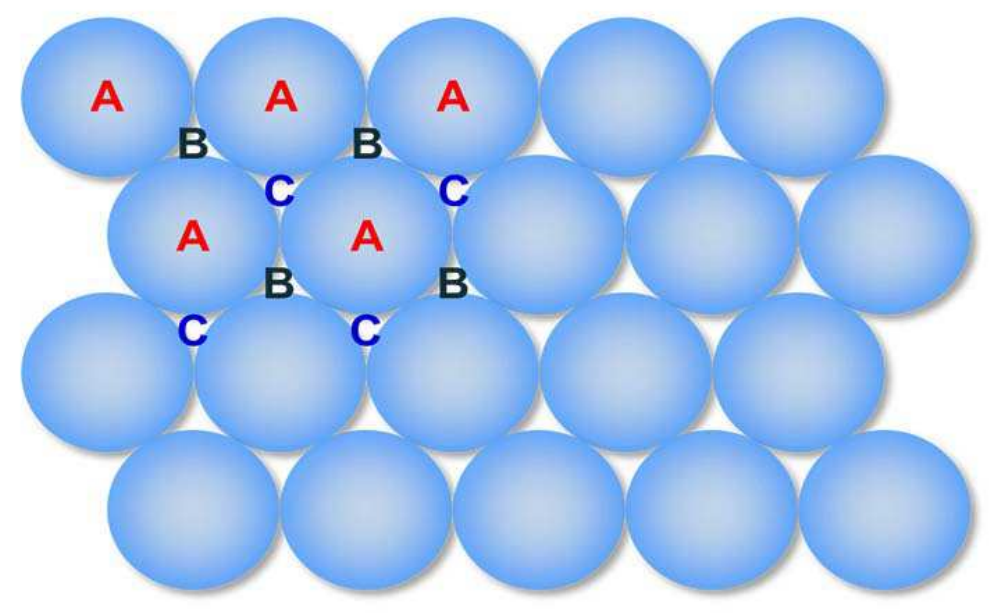

Fig. 1. A close-packed layer of spheres occupying positions A. A second and identical layer of spheres can be placed on top of this layer and occupy positions B or C.

Figure 2 shows the structure of an S block. Figure 2(b) shows a structure with oxygen layers only, and Figure 2(a) shows the top oxygen layer when viewed from above. For a better presentation, the ratio of the oxygen ion diameter to the oxygen-oxygen distance is set to be much smaller than it actually is. Figure 2(a) shows a $60^{\circ}$ rhombus consisted of close-packed oxygen ions; and the structure in Fig. 2(b) clearly shows that an S block is built from the stacking of close-packed oxygen layers in an "ABCABC..." sequence. It is important to note that an $S$ block consists of only two oxygen layers, although three layers are shown in Fig. 2(b). For example, one can consider an $S$ block consisting of the top and middle oxygen layers, with the bottom layer belonging to the underneath block. Therefore, one can see that each $\mathrm{S}$ block contains eight oxygen ions, with four from each layer.

Figure 2(c) shows a structure with both oxygen ions and cations. The small solid circles show the cations on tetrahedral sites, while the small open circles show the cations on octahedral sites. Between the top and middle oxygen layers, there are one cation at an octahedral site and five cations at tetrahedral sites. The octahedral site is formed by three 

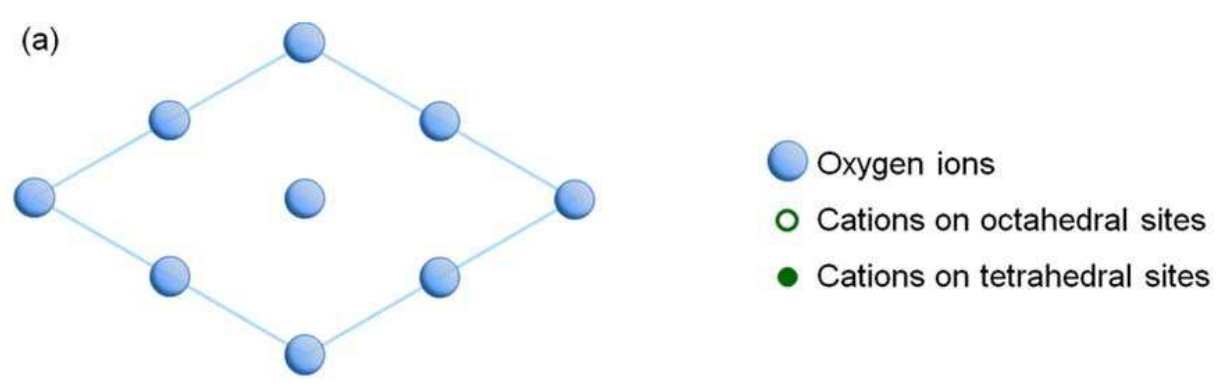

(b)

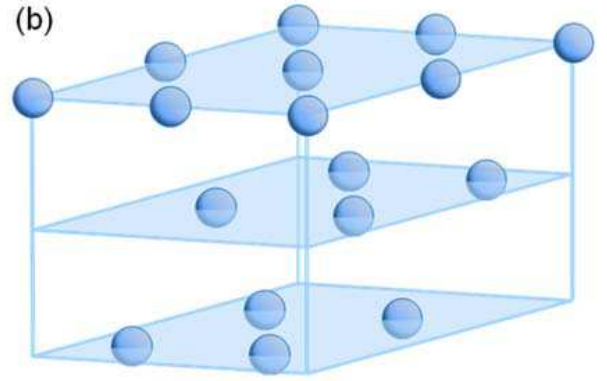

(c)

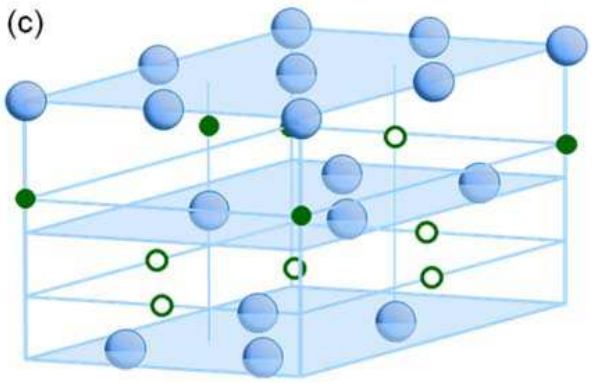

Fig. 2. Structure of S block. (a) Top oxygen layer viewed from above. (b) S block structure with oxygen layers only. (c) S block structure with both oxygen ions and cations.

oxygen ions in the top layer and three in the middle layer and, therefore, lies halfway between the top and middle oxygen layers. Among five tetrahedral cations, one occupies the tetrahedral site formed by three oxygen ions in the top layer and another in the middle layer; the other four occupy the corners of a $60^{\circ}$ rhombus, with each shared by four $60^{\circ}$ rhombuses. Overall, between the top and middle layers, there are one cation at an octahedral site and two cations at tetrahedral sites.

Between the middle and bottom oxygen layers, there are five cations at the octahedral sites, all of which are within a $60^{\circ}$ rhombus halfway between the oxygen layers. Among these five cations, four are at the middle points of four rhombus sides, with each shared by two rhombuses; and the other is at the center of the rhombus and is not shared by any other rhombuses. Overall, there are three octahedral cations between the middle and bottom oxygen layers.

In total, each $S$ block contains eight oxygen ions in close-packed plans, four cations at octahedral sites, and two cations at tetrahedral sites. If the cations are iron ions, the block contains two formula units of $\mathrm{Fe}_{3} \mathrm{O}_{4}$.

Three points should be noted about $S$ blocks. First, the hexagonal structure of the $S$ block is clearly shown in Fig. 2. The vertical axis of the structure is referred to as the $c$ axis. Second, the $\mathrm{S}$ block is often referred to as a spinel block. This is because the oxygen ions and cations are so distributed that they form precisely the cubic spinel arrangement with the [111] axis 
vertical. Third, among the three types of fundamental blocks which make up hexagonal ferrites, the $\mathrm{S}$ block is the smallest one and is the only one containing no barium ions.

Figure 3 shows the structure of an R block. Figure 3(b) shows a structure with oxygen and barium ions only. Figure 3(a) shows the top layer of the structure when viewed from above. Figures 3(a) and 3(b) clearly show that an R block consists of three close-packed oxygen layers, with one oxygen ion in the middle layer replaced by a barium ion $\mathrm{Ba}^{2+}$. These layers are stacking on each other in an "ABAB..." sequence. The top, middle, and bottom layers contain four, three, and four oxygen ions, respectively. Overall, each R block contains eleven oxygen ions and one barium ion.

Figure 3(c) shows a structure with all ions. The small solid circles show four cations which occupy the trigonal sites in the middle layer. As each trigonal site is shared by four $60^{\circ}$ rhombuses, only one of these four cations belongs to the structure unit shown. The small open circles show five cations at octahedral sites. Among the five sites, one lies halfway between the top and middle oxygen layers, one lies halfway between the middle and bottom oxygen layers, and three are underneath the bottom oxygen layer.
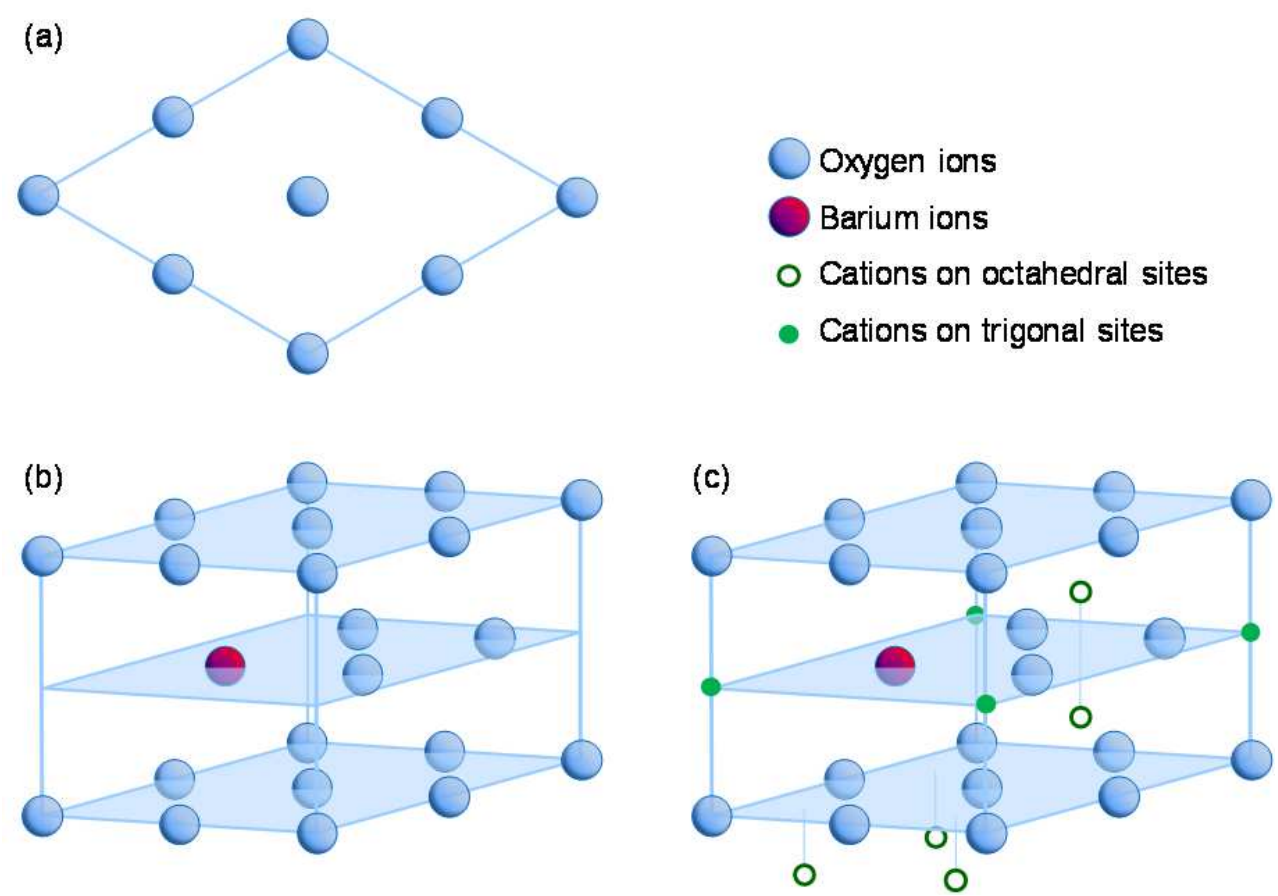

Fig. 3. Structure of R block. (a) The top oxygen layer viewed from above. (b) R block structure with oxygen and barium ions only. (c) R block structure with all ions.

In total, each $\mathrm{R}$ block contains eleven oxygen ions, one barium ion, one cation in a trigonal site, and five cations in octahedral sites. If those cations are iron ions, one can denote an $\mathrm{R}$ block by $\mathrm{BaFe}_{6} \mathrm{O}_{11}$. Note that the $\mathrm{R}$ block has an hcp structure thanks to the "ABAB..." stacking sequence; the $c$ axis of the structure is along the vertical axis. 
It should be noted that both $2 \mathrm{Fe}_{3} \mathrm{O}_{4}$ (S block) and $\mathrm{BaFe}_{6} \mathrm{O}_{11}$ ( $\mathrm{R}$ block) are not electrically neutral if the iron ions are trivalent. This, however, is not a problem, because hexagonal ferrites are always made up of more than one type of block. For example, the combination of one $\mathrm{S}$ block and one $\mathrm{R}$ block yields $\mathrm{BaFe}_{12} \mathrm{O}_{19}$ which is indeed electrically neutral when the iron ions are $\mathrm{Fe}^{3+}$.

\subsection{Structure and static magnetic properties of BaM materials}

The M-type barium hexagonal ferrite, often called $\mathrm{BaM}$, has a chemical formula of $\mathrm{BaFe}_{12} \mathrm{O}_{19}$, with all of the iron ions being trivalent. The crystal structure of BaM is the same as that of the mineral magnetoplumbite. Each elementary cell is formed by the stacking of $S, R, S^{*}$, and $\mathrm{R}^{*}$ blocks and, therefore, contains ten layers of close-packed oxygen ions. Among these ten layers, two layers contain barium ions, as shown by the middle layer in the structure in Fig. $3(b)$. The two layers within the $S$ (or $S^{*}$ ) block, the layer right above the block, and the layer right underneath the block are stacked in an "ABCABC..." sequence. The three layers within the $\mathrm{R}$ (or $\mathrm{R}^{*}$ ) block, the layer right above the block, and the layer right underneath the block are stacked in an "ABAB..." sequence.

The distribution of the iron ions in the BaM lattice sites and the orientation of their magnetic moments are summarized in Table II. A detailed description of these sites is given in Section 2.1. Magnetically, ferrite materials have majority and minority sublattices. Within each sublattice, the magnetic moments are parallel to each other. The moments in two sublattices, however, are opposite to each other. The difference between the total moments of two magnetic sublattices determines the saturation magnetization of the material. In Table II, an upward-directed arrow indicates a contribution to the majority magnetic sublattice, while a downward-directed arrow indicates a contribution to the minority magnetic sublattice.

\begin{tabular}{c|c|c|c|cc}
\hline Block & Formula & Tetrahedral & Octahedral & Trigonal & Net \\
\hline $\mathrm{S}$ & $2 \mathrm{Fe}_{3} \mathrm{O}_{4}$ & $2 \downarrow$ & $4 \uparrow$ & - & $2 \uparrow$ \\
\hline $\mathrm{R}$ & $\mathrm{BaFe}_{6} \mathrm{O}_{11}$ & - & $3 \uparrow, 2 \downarrow$ & $1 \uparrow$ & $2 \uparrow$ \\
\hline $\mathrm{S}^{*}$ & $2 \mathrm{Fe}_{3} \mathrm{O}_{4}$ & $2 \downarrow$ & $4 \uparrow$ & - & $2 \uparrow$ \\
\hline $\mathrm{R}^{*}$ & $\mathrm{BaFe}_{6} \mathrm{O}_{11}$ & - & $3 \uparrow, 2 \downarrow$ & $1 \uparrow$ & $2 \uparrow$ \\
\hline
\end{tabular}

Table II. Distributions of $\mathrm{Fe}^{3+}$ ions in a unit cell of BaM materials ${ }^{44,45}$

The data in Table II indicates that a full unit cell of BaM materials contains two formula units of $\mathrm{BaFe}_{12} \mathrm{O}_{19}$; the net magnetic moment in each unit cell is equal to the moment of eight $\mathrm{Fe}^{3+}$ ions. The magnetic moment of each $\mathrm{Fe}^{3+}$ ion is usually taken as $5 \mu_{\mathrm{B}}$. As a result, each unit cell of $\mathrm{BaM}$ is expected to have a net magnetic moment of $40 \mu_{\mathrm{B}}$. One can define the vertical axis of the building blocks as the $c$ axis of the unit cell and one of the sides of the $60^{\circ}$ oxygen rhombus (see Fig. 2(a)) as the $a$ axis. In these terms, the lengths of the $c$ and $a$ axes of a BaM unit cell are about $23.2 \AA$ and $5.89 \AA$, respectively. With these parameters, one can estimate the saturation induction $4 \pi M_{\mathrm{s}}$ of $\mathrm{BaM}$ as about $6680 \mathrm{G}$. This value is close to the $4 \pi M_{\mathrm{s}}$ value measured at low temperatures. ${ }^{45}$ At room temperature, BaM bulk crystals usually have a $4 \pi M_{\mathrm{s}}$ value of about $4700 \mathrm{G} ; 47,48$ while BaM thin films usually show a slightly smaller value.

BaM materials have uniaxial magneto-crystalline anisotropy, with the easy axis along the $c$ axis of the hexagonal structure. The effective anisotropy field $H_{\mathrm{a}}$ is about $17 \mathrm{kOe} .{ }^{45}$ This field 
is three orders of magnitude higher than that in YIG materials. It is this strong built-in field that facilitates ferromagnetic resonances in BaM materials at mm-wave frequencies with no need of large external bias fields and, thereby, makes the research field of BaM materials and devices very attractive and promising.

Table III gives the room-temperature structure and physical properties of BaM crystals as well as the Curie temperature of BaM materials.

\begin{tabular}{l|l|l}
\hline Parameter & Value & Reference \\
\hline Lattice constant $c$ & $23.2 \AA$ & {$[45]$} \\
\hline Lattice constant $a$ & $5.89 \AA$ & {$[45]$} \\
\hline X-ray density & $5.28 \mathrm{~g} / \mathrm{cm}^{3}$ & {$[44]$} \\
\hline Curie temperature $T_{c}$ & $725 \mathrm{~K}$ & {$[45]$} \\
\hline Exchange energy parameter $A_{\mathrm{ex}}$ & $6.4 \times 10^{-7} \mathrm{erg} / \mathrm{cm}$ & {$[7]$} \\
\hline Saturation induction $4 \pi M_{s}$ & $4700 \mathrm{G}$ & {$[47],[48]$} \\
\hline Effective anisotropy field $H_{\mathrm{a}}$ & $17 \mathrm{kOe}$ & {$[45]$} \\
\hline Damping constant $\alpha$ & $7 \times 10^{-4}$ & {$[7]$} \\
\hline
\end{tabular}

Table III. Properties of M-type barium hexagonal ferrites

\subsection{Ferromagnetic resonances in BaM materials}

One typically makes use of ferromagnetic resonance (FMR) techniques to characterize microwave and mm-wave losses in ferrite materials. Figure 4 shows the FMR effect. Figure 4(a) gives a schematic presentation of the FMR operation, where the magnetization $M$ absorbs energy from the microwave magnetic field $h$ and maintains a fixed angle of precession around the static magnetic field $H$. The FMR effect manifests itself in a peak response in the measurement of the microwave power absorption in the material as a function of the static magnetic field, as shown in Fig. 4(b). The full width at the half maximum of this so-called FMR absorption curve is usually taken as the FMR linewidth $\Delta H_{\mathrm{FMR}}$. The origin of the FMR linewidth differs significantly in different materials. In ferrites, typical relaxation processes that contribute to $\Delta H_{\mathrm{FMR}}$ include magnon-phonon scattering, two-magnon scattering, charge transfer relaxation, and processes associated with slowly relaxing impurity and rapidly relaxing impurity.49,50 The identification and quantization of each process demand extensive measurements and numerical analyses. For this reason, one typically uses $\Delta H_{\mathrm{FMR}}$ as a measure of the overall loss of the material. Very often, FMR measurements are carried out with field modulation and lock-in detection techniques, and the actual FMR data consist of the derivative of the power absorption curve, as shown in Fig. 4(c). In this case, one measures the peak-to-peak FMR linewidth $\Delta H_{\mathrm{pp}}$. If the FMR profile is Lorentzian in shape, one can convert $\Delta H_{\mathrm{pp}}$ into $\Delta H_{\mathrm{FMR}}$ simply by multiplying $\Delta H_{\mathrm{pp}}$ by $\sqrt{3}$. One can also conduct FMR measurements by keeping the field constant and sweeping the frequency. The detail on the conversion between the field and frequency linewidths is given in Section 4.1.

One usually measures FMR responses in BaM films with the application of an external magnetic field $H$ along the film easy axes. For a BaM film with the $c$ axis out of the plane, the magnetic torque equation yields an FMR frequency of 


$$
f_{\mathrm{FMR}}=|\gamma|\left(H+H_{a}-4 \pi M_{s}\right)
$$

where $|\gamma|=2.8 \mathrm{MHz} / \mathrm{Oe}$ is the absolute value of the gyromagnetic ratio. For a film with the $c$ axis in the plane, the FMR frequency is given by

$$
f_{\mathrm{FMR}}=|\gamma| \sqrt{\left(H+H_{a}\right)\left(H+H_{a}+4 \pi M_{s}\right)}
$$
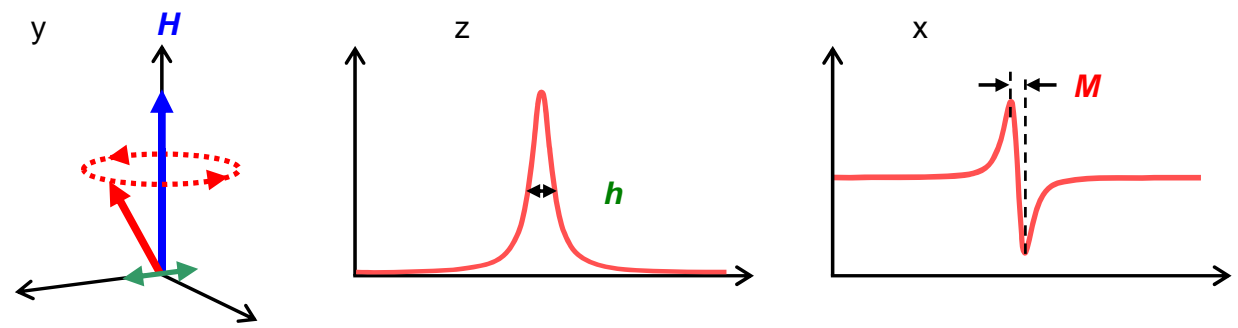

Fig. 4. Schematic presentation of ferromagnetic resonance (FMR)

The films prepared with different processes can show significantly different FMR linewidth values. The lowest linewidth so far was reported by Song et al. for a BaM film grown by PLD techniques. ${ }^{7}$ This film had an out-of-plane $c$-axis orientation and was $0.85 \mu \mathrm{m}$ thick. The film showed a $60.3 \mathrm{GHz}$ FMR linewidth $\Delta H_{\mathrm{FMR}}$ of $27 \mathrm{Oe}$, which matched the value of singlecrystal BaM bulk materials. The frequency-dependent FMR measurements yielded a linear response with a slope of $0.5 \mathrm{Oe} / \mathrm{GHz}$, which corresponded to an effective damping constant $\alpha$ of about $7 \times 10^{-4}$.

\section{Development of M-type barium hexagonal ferrite films}

This section reviews the recent advances made in the development of BaM film materials. The section consists of four subsections, each on a separate effort. The four efforts are (1) the development of BaM thin films that have both low losses and high remanent magnetizations, (2) the deposition of BaM thin films on metallic electrodes, (3) the growth of $\mathrm{BaM}$ thin films on semiconductor substrates, and (4) the development of BaM thick films on semiconductor substrates. The motivations and implications of each effort are described in each subsection.

\subsection{Development of low-loss, self-biased BaM thin films}

In terms of device applications, BaM films with narrow FMR linewidths $\left(\Delta H_{\mathrm{FMR}}\right)$ and high remanent magnetizations $\left(M_{\mathrm{r}}\right)$ are very desirable. The narrow linewidth is critical for the realization of low insertion losses for certain devices, while the high remanent magnetization facilitates the operation of devices in absence of external magnetic bias fields, namely, self-biased operation. As mentioned above, Song et al. succeeded in the PLD growth of BaM thin films that showed an FMR linewidth as narrow as single-crystal BaM bulks.7 These films, however, showed a remanent magnetization much smaller than the saturation magnetization $\left(M_{\mathrm{s}}\right)$. The main reason for this small remanent magnetization lies on the out- 
of-plane $c$-axis orientation. Such $c$-axis orientation gives rise to a near unity demagnetizing factor along the film normal direction and a corresponding small $M_{\mathrm{r}}$ value.

Yoon et al. were able to use PLD techniques to grow in-plane $c$-axis oriented BaM films with higher remanent magnetizations, at an $M_{\mathrm{r}} / M_{\mathrm{s}}$ ratio of about $0.94 .8,9$ Those "in-plane" films, however, had very broad FMR peaks, with a $50-60 \mathrm{GHz}$ peak-to-peak FMR linewidth $\Delta H_{\mathrm{pp}}$ of about 1150 Oe or larger. Note that the high $M_{\mathrm{r}} / M_{\mathrm{s}}$ ratios in those films derived from the near zero demagnetizing factor along the in-plane $c$ axis. Song et al. reported "in-plane" BaM films with slightly lower $M_{\mathrm{r}} / M_{\mathrm{s}}$ ratios at about 0.84 , but with much narrower peak-to-peak linewidths of about 250 Oe. ${ }^{35}$ Those films, however, were made through a hybrid process that involved both PLD and LPE methods along with post-deposition surface flux cleaning.

In 2010, Song et al. reported the development of in-plane $c$-axis oriented BaM thin films that had both small $\Delta H_{\mathrm{FMR}}$ and high $M_{\mathrm{r}}{ }^{10}$ The films were grown on an a-plane sapphire substrate by basic PLD techniques. The high quality was realized through several changes in the substrate temperature during the deposition, along with the optimization of other PLD control parameters. The sequential changes in substrate temperature resulted in a series of BaM layers with slightly different structure properties. This quasi-multi-layered configuration served to release interfacial strain and thereby realize high-quality films. The films showed a $M_{\mathrm{r}} / M_{\mathrm{s}}$ ratio that is higher than any previous BaM films, an FMR linewidth that is a factor of four smaller than those of previous PLD films, and an effective anisotropy field that closely matches the value of BaM bulk crystals.

Figure 5 gives the $x$-ray diffraction (XRD) data for one of those BaM films. Figure 5(a) shows the intensity vs. angle profile, and Figure 5(b) shows an XRD rocking curve. The profile in Fig. 5(a) shows three strong peaks. The central peak comes from the sapphire, while the other two are from the $m$ planes of the BaM film. There are no peaks for other planes of the BaM film or the sapphire substrate, or for other phases. The rocking curve for the $\mathrm{M}(200)$ peak in Fig. 5(b) shows a "full width at half maximum" (FWHM) of about $0.85^{\circ}$. This value is very small and indicates a very small deviation of the c-axis orientation over the film. Note that the FWHM value is about 10\% lower than that reported in Ref. [35]. These results clearly indicate that the film has a $c$-axis that is in the plane of the film and is highly oriented.

(a)

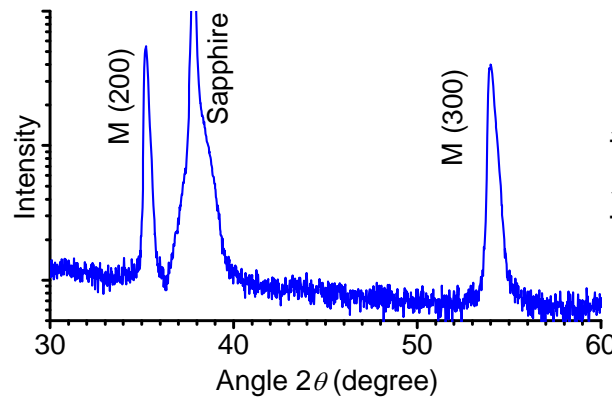

(b)

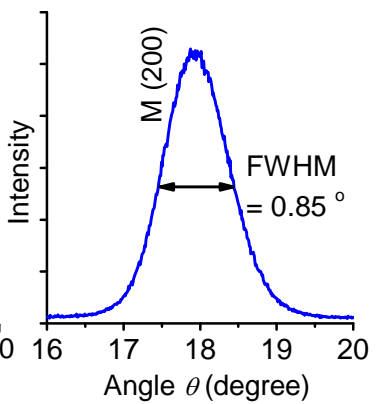

Fig. 5. (a) X-ray diffraction spectrum of a BaM film. (b) X-ray diffraction rocking curve of the same film. (After Ref. [10]) 
Figure 6 shows two scanning electron microscopy (SEM) images. The one in Fig. 6(a) is for the film surface, while the one in Fig. 6(b) is for the cross section of the film. The image in Fig. 6(a) shows a reasonably smooth surface and no notable holes. The image also shows many fine lines, as indicated by the black arrows. These lines correspond to fine parallel cracks along the direction perpendicular to the $c$ axis. The parallelism of these cracks gives a rough measure of the good orientation of the $c$ axis. The image in Fig. 6(b) shows that the film thickness is uniform at $2.52 \mu \mathrm{m}$ and that there are no cracks at the film-substrate interface.
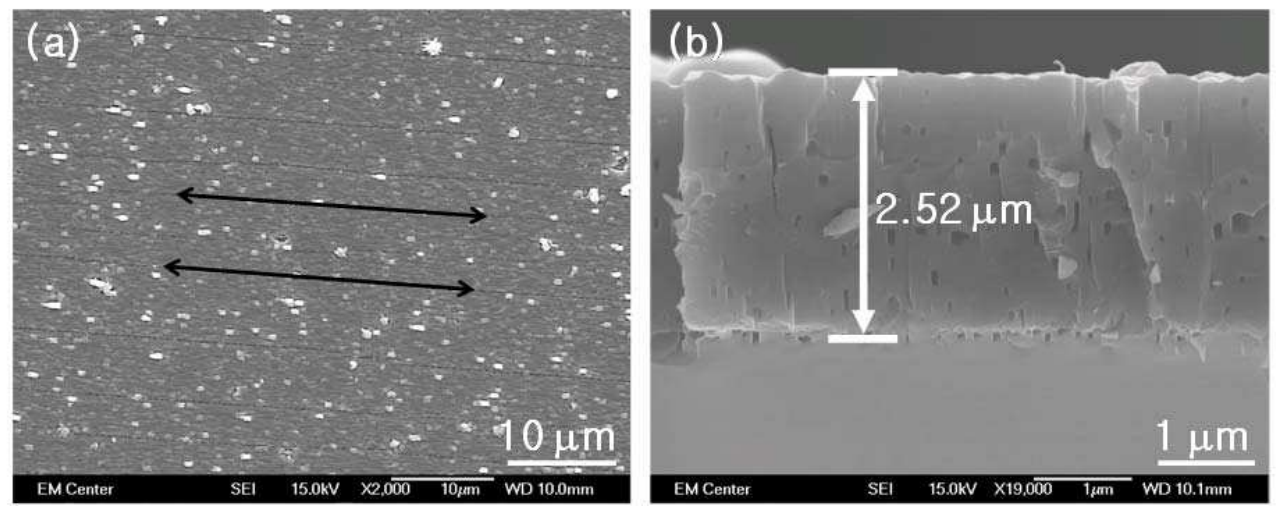

Fig. 6. Scanning electron microscopy images of a BaM film. (a) Film surface. (b) Film cross section. (After Ref. [10])

Figure 7 shows two magnetic induction $(4 \pi M)$ vs. magnetic field $(H)$ hysteresis loops measured with the fields applied along the in-plane easy and hard axes, as indicated. The easy axis is along the $c$ axis defined by the XRD measurements. The dashed lines indicate the extrapolations used to determine the effective anisotropy field $H_{\mathrm{a}}$. Three results are evident in Fig. 7. (1) The film has an extremely well defined in-plane uniaxial anisotropy with the easy axis along the $c$ axis. (2) The data indicate an anisotropy field $H_{\mathrm{a}}$ of about 16.9 $\mathrm{kOe}$, a saturation induction $4 \pi M_{\mathrm{s}}$ of about $3.9 \mathrm{kG}$, and an easy-axis coercive force of about 200 Oe. These values are close to those for high-quality BaM films as reported previously.7,35 (3) The film has an $M_{\mathrm{r}} / M_{\mathrm{s}}$ ratio of 0.99 , which is very close to unity and is the highest value ever obtained for BaM films. These results clearly confirm the in-plane orientation of the $c$ axis and demonstrate the near ideal in-plane uniaxial anisotropy for this film.

Figure 8 gives representative FMR data. Figure 8(a) shows three FMR absorption derivative profiles at different frequencies, as indicated. The circles show the data, and the curves show fits to a Lorentzian derivative trial function. The lines and arrows indicate the linewidths based on these fits. The circles in Fig. 8(b) show the measured FMR field vs. frequency data. The line shows theoretical FMR fields based on Eq. (2) and the $H_{\mathrm{a}}$ and $4 \pi M_{\mathrm{s}}$ values cited above. These data show four results. (1) The film has a narrow FMR linewidth. The values indicated are four times lower than those for previous PLD films. ${ }^{8,9}$ (2) There is a very good match between the experimental FMR profiles and the Lorentzian fits. (3) The theoretical FMR fields match nicely with the experimental values. This match confirms the $H_{\mathrm{a}}$ and $4 \pi M_{\mathrm{s}}$ values obtained from the hysteresis loop measurements. (4) The FMR frequency-field curve 


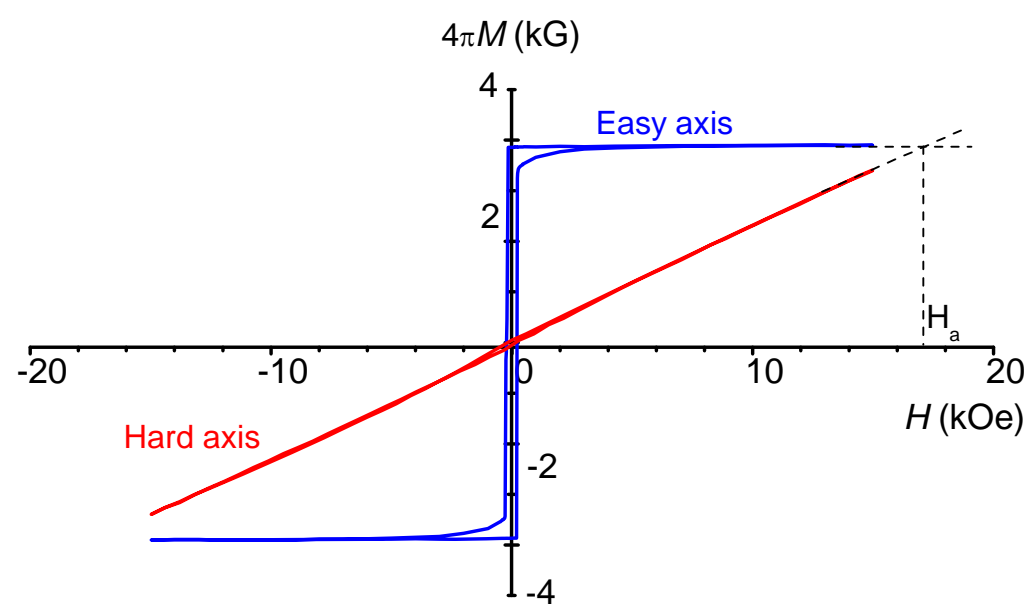

Fig. 7. Magnetic induction $4 \pi M$ - field $H$ loops of a BaM film measured with the fields along the in-plane easy and hard axes. The dashed lines indicate the determination of the anisotropy field $H_{\mathrm{a}}$. (After Ref. [10])

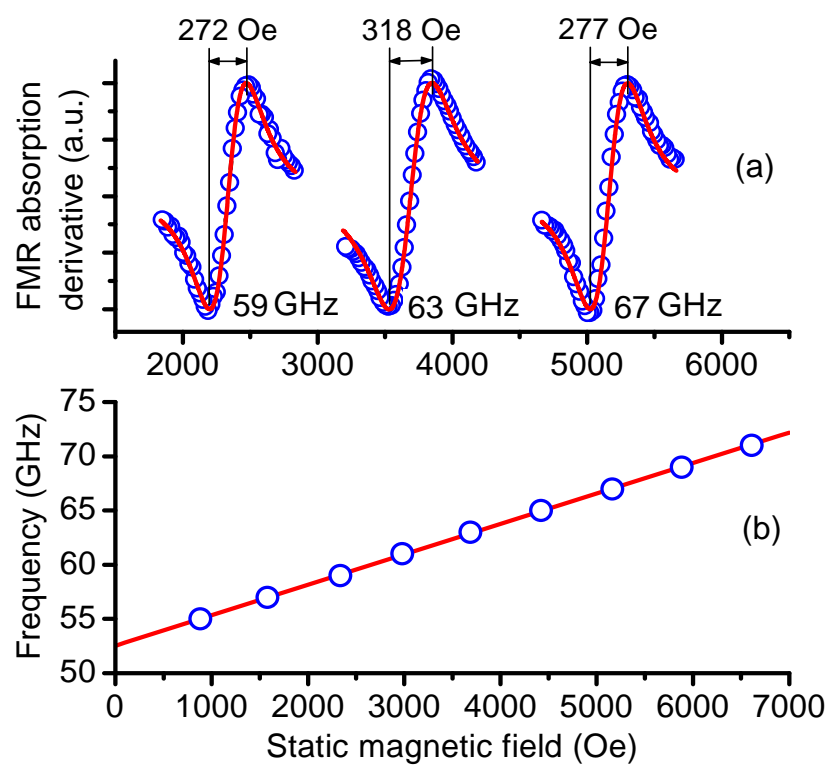

Fig. 8. FMR results for a BaM film. (a) FMR absorption derivative profiles measured at different frequencies. (b) FMR frequency as a function of static magnetic field. In both (a) and (b), the open circles show the data. The curves in (a) show Lorentzian fits. The line in (b) shows theoretical results. (After Ref. [10]) 
shows an almost linear response. This linear dependence results from the fact that the anisotropy field $H_{\mathrm{a}}$ is significantly larger than the induction $4 \pi M_{\mathrm{s}}$.

These results clearly demonstrate the feasibility of the basic PLD growth of BaM films with both high remanent magnetizations and low losses. Future work on the development of BaM films with similar quality but different anisotropy fields is of great interest. This can be realized, for example, through Sc or $\mathrm{Al}$ doping in BaM films. ${ }^{51,52}$

\subsection{Deposition of BaM thin films on metallic electrodes}

Single-crystal sapphire $\left(\alpha-\mathrm{Al}_{2} \mathrm{O}_{3}\right)$ has been the substrate of choice for the growth of $\mathrm{BaM}$ films by PLD and sputtering.7,8,9,10,31,35,36 Sapphire is chosen because it has a rhombohedral crystal structure $\left(a=5.128 \AA\right.$ and $\left.\alpha=55^{\circ} 22^{\prime}\right)$ which is close to the hexagonal structure of $\mathrm{BaM} ;{ }^{31}$ and the mismatches of the lattice parameters and thermal expansion coefficients between sapphire and BaM materials are relatively small.7,31,36 Certain device applications, however, require the growth of BaM films on conductive substrates. In coupled-line and stripline-type devices, for example, a ground plane is needed underneath the active layer.

In 2010, Nie et al. succeeded in the fabrication of out-of-plane $c$-axis oriented BaM thin films on platinum $(\mathrm{Pt})$ electrodes through metallo-organic decomposition (MOD) techniques. 15 The films were prepared on $300 \mathrm{~nm}$-thick (111)-oriented Pt layers which were sputtered on Si wafers. The fabrication processes include three main steps, (1) spin coating of a precursor onto a substrate, (2) annealing at different temperatures $\left(150-450{ }^{\circ} \mathrm{C}\right)$ to remove solvents and realize metallo-organic decomposition, and (3) rapid thermal annealing (RTA) at high temperatures $\left(850-900{ }^{\circ} \mathrm{C}\right)$ to facilitate the formation of a proper structure. It was found that the RTA step was very critical for the realization of high-quality BaM films. It was demonstrated, for example, that the temperature significantly affects the crystalline structure of the film, and the type of the process gas strongly affects both the remanent magnetization and FMR linewidth.

Figure 9 shows the magnetic moment vs. field hysteresis loops measured for three film samples for both in-plane $(||)$ and out-of-plane $(\perp)$ fields. The films were obtained with different RTA processes. For films \#1 and \#2, the RTA processes were done in $\mathrm{O}_{2}$ and $\mathrm{N}_{2}$, respectively. For film \#3, the RTA process was made in $\mathrm{N}_{2}$ first and then in $\mathrm{O}_{2}$. The data for films \#1, \#2, and \#3 are shown in Figs. 9(a), 9(b), and 9(c), respectively. One can clearly see that (1) these films show significantly different magnetic properties and (2) film \#3 has the highest out-of-plane remanent magnetization, with $M_{\mathrm{r}} / M_{\mathrm{s}} \approx 0.93$, and the lowest out-of-plane coercivity, with $H_{\mathrm{c}}=4.5 \mathrm{kOe}$. Note that high remanent magnetizations are desirable from the point of view of device applications, as discussed in Section 3.1.

Nie et al. reported that film \#3 not only showed the largest $M_{\mathrm{r}} / M_{\mathrm{s}}$ ratio, but also had the lowest loss. Figure 10 presents the FMR linewidth vs. frequency data obtained for film \#3. One can see that the film had a linewidth of about 338 Oe at $60 \mathrm{GHz}$ and a slope of 4.2 $\mathrm{Oe} / \mathrm{GHz}$ over the $45-60 \mathrm{GHz}$ range. This slope corresponds to an effective damping constant $\alpha$ of about 0.006 . These values are about one order of magnitude larger than those of the outof-plane $c$-axis oriented BaM films grown by PLD on sapphire substrates. ${ }^{7}$ Nevertheless, they are reasonably low in terms of practical applications. 


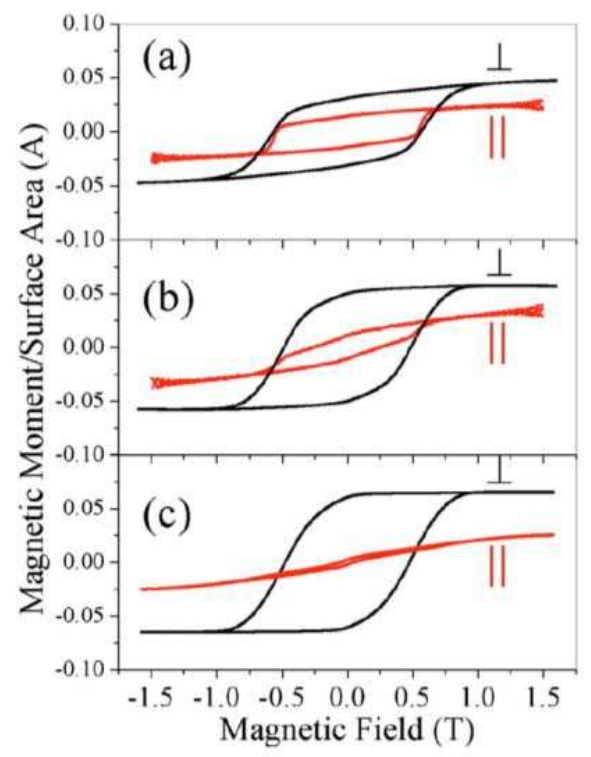

Fig. 9. Magnetic hysteresis loops obtained for three BaM films for both in-plane $(||)$ and out-of-plane $(\perp)$ external magnetic fields. (After Ref. [15])

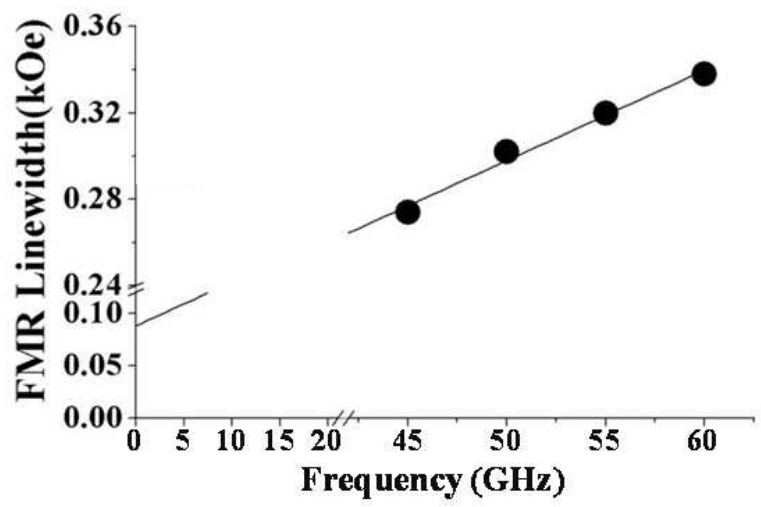

Fig. 10. FMR linewidth vs. frequency data for a BaM film. (After Ref. [15])

Two points should be highlighted. First, the work by Nie et al. clearly demonstrated the feasibility of the use of MOD techniques to fabricate on metallic electrodes BaM thin films with high $M_{\mathrm{r}} / M_{\mathrm{s}}$ ratios and reasonable low losses.15 Second, in comparison with PLD techniques, the MOD techniques are relatively inexpensive and can be used to make relatively large films. It should also be noted that for the data shown in Figs. 9 and 10, the films are about 200-500 nm thick. Future work on the use of MOD to make much thicker $\mathrm{BaM}$ films is of great interest. In principle, this can be done by the repetition of those processing steps mentioned above. Future work on the fabrication of BaM films on other "more conventional" electrodes, such as copper, is also of great interest. Note that the 
deposition of BaM films on copper can be challenging because one might need to face the issues associated with copper diffusion or oxidation at high temperatures.

\subsection{Deposition of BaM Thin films on semiconductor substrates}

The growth of high-quality BaM films on semiconductor substrates can allow for the development of BaM-based devices that are compatible with monolithic integrated circuits. In 2006, Chen et al. succeeded in the deposition of BaM thin films on single-crystal $6 \mathrm{H}-\mathrm{SiC}$ substrates by PLD techniques.11 The $6 \mathrm{H}-\mathrm{SiC}$ substrates were chosen not only because they are promising wide band-gap semiconductor materials for next-generation electronics, but also because they have the same hexagonal crystal structure as the BaM materials, with a lattice mismatch of $4.38 \%$ in the $c$ plane. ${ }^{13}$ The films showed good crystal textures and effective anisotropy fields higher than $15 \mathrm{kOe}$. The loss of the films, however, was very high, with $\Delta H_{\mathrm{pp}}>1 \mathrm{kOe}$. Such broad linewidths resulted from the random orientation of the grains in the BaM films and the diffusion of silicon from the substrates into the BaM films. Through the introduction of an interwoven $\mathrm{MgO} / \mathrm{BaM}$ multilayered buffer into the film-substrate interface, Chen et al. were able to significantly improve the quality of the films and reduce the $\Delta H_{\mathrm{pp}}$ value down to 500 Oe at $55 \mathrm{GHz} \cdot{ }^{12}$ The $\mathrm{MgO} / \mathrm{BaM}$ multilayered buffer was also grown by PLD techniques. This buffer not only reduced the lattice mismatch between the film and the substrate, but also suppressed silicon diffusion at high temperatures.

In 2007, Chen et al. made use of a hybrid process and succeeded in the deposition of BaM films on $6 \mathrm{H}-\mathrm{SiC}$ substrates with even lower losses. ${ }^{13}$ The process involved three steps, (1) growth of an $\mathrm{MgO}$ (111) buffer layer on a $6 \mathrm{H}-\mathrm{SiC}$ (0001) single crystal wafer by the MBE method, (2) growth of a BaM film onto the MgO layer by the PLD techniques, and (3) postannealing in air. The $\mathrm{MgO}$ layer was grown at a substrate temperature of $150{ }^{\circ} \mathrm{C}$. The BaM film was deposited at a substrate temperature of $915{ }^{\circ} \mathrm{C}$. The post-annealing was done at $1050{ }^{\circ} \mathrm{C}$. The thickness of the $\mathrm{MgO}$ layers varied from $2 \mathrm{~nm}$ to $12 \mathrm{~nm}$, and the BaM films were $400 \mathrm{~nm}$ thick. The samples showed an abrupt $\mathrm{MgO} / \mathrm{BaM}$ interface, with no Si or Fe diffusions. The films had out-of-plane $c$-axis orientation and showed an out-of-plane uniaxial anisotropy. The effective anisotropy field and saturation induction were $16.9 \mathrm{kOe}$ and $4400 \mathrm{G}$, respectively, which were both close to the values for single-crystal BaM bulks (see Table III). The FMR measurements on these films indicated linewidth that were significantly narrower than those of previous $\mathrm{BaM}$ films grown on $6 \mathrm{H}-\mathrm{SiC}$ substrates.11,12 Before the post-annealing, the films showed a $\Delta H_{\text {pp }}$ value of about 220 Oe at $53 \mathrm{GHz}$. After the post-annealing, this value was reduced to about 100 Oe. Figure 11 shows an FMR profile measured at $53 \mathrm{GHz}$ for a BaM film sample where the $\mathrm{MgO}$ buffer layer is $10 \mathrm{~nm}$ thick. This profile indicates a $\Delta H_{\mathrm{pp}}$ value of 96 Oe.

The samples discussed above involved the MBE growth of $\mathrm{MgO}$ buffer layers and the PLD growth of BaM films. In 2010, Cai et al. demonstrated the MBE growth of both MgO buffer layers and $\mathrm{BaM}$ thin films on $6 \mathrm{H}-\mathrm{SiC}$ substrates. ${ }^{14}$ The $\mathrm{MgO}$ layers were grown at a substrate temperature of $150{ }^{\circ} \mathrm{C}$. For the growth of $\mathrm{BaM}$ films, the substrate temperature was set to $800{ }^{\circ} \mathrm{C}$. The oxygen pressure was optimized to allow for the growth of BaM films with nearperfect structures. As in Ref. [13], the $6 \mathrm{H}-\mathrm{SiC}$ substrates were single-crystal (0001) wafers, the $\mathrm{MgO}$ layers had (111) orientation, and the BaM $c$ axis was out-of-plane. Figure 12 shows the induction vs. field hysteresis loops obtained for a $200 \mathrm{~nm}$-thick BaM film grown on a 


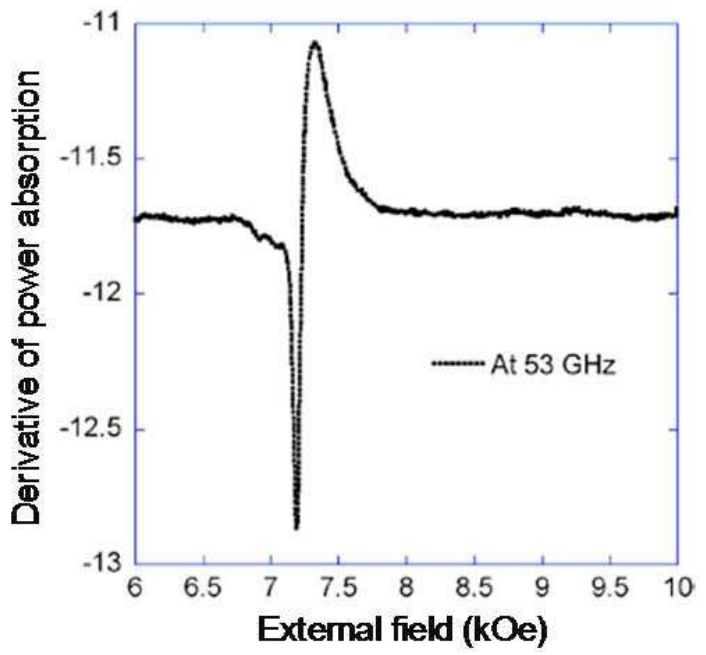

Fig. 11. FMR power absorption derivative profile for a BaM thin film grown on a singlecrystal $6 \mathrm{H}-\mathrm{SiC}$ substrate. (After Ref. [13])

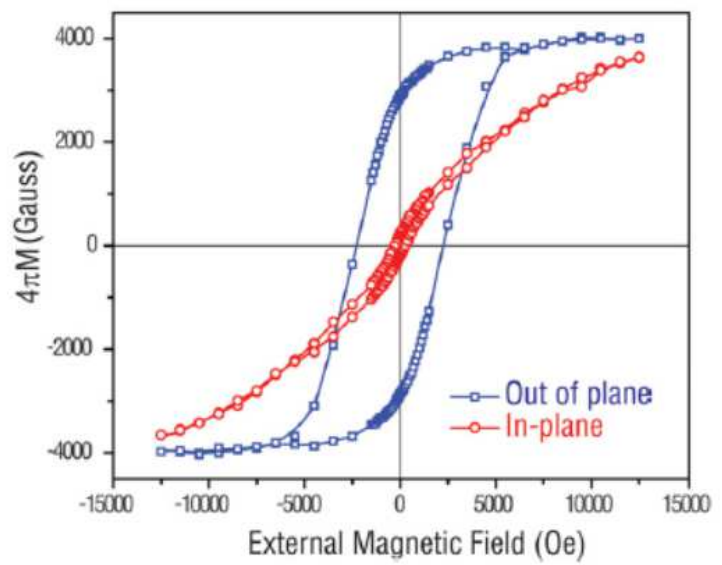

Fig. 12. Hysteresis loops for a BaM thin film grown on a single-crystal $6 \mathrm{H}-\mathrm{SiC}$ substrate by the MBE method. The squares and circles show the data for external fields applied out of the film plane and in the film plane, respectively. (After Ref. [14])

$6 \mathrm{H}-\mathrm{SiC}$ substrate with a $10 \mathrm{~nm}$-thick $\mathrm{MgO}$ buffer layer. The data confirm the out-of-plane anisotropy of the film and indicate an effective anisotropy field of $16.2 \mathrm{kOe}$, a saturation induction of $4100 \mathrm{G}$, and a $M_{\mathrm{r}} / M_{\mathrm{s}}$ ratio of 0.7 . These results, together with X-ray photoelectron spectroscopy and atomic force microscopy results, clearly indicate the feasibility of the use of MBE to prepare high-quality BaM thin films on $6 \mathrm{H}-\mathrm{SiC}$ substrates.

There are two points to be mentioned. First, the low-loss films reported in Ref. [13] had very low remanent magnetizations. Future work on the use of the same process to grow in-plane 
$c$-axis oriented BaM films is of great interest. Such films have a near zero demagnetizing factor along the in-plane $c$ axis and, therefore, are expected to exhibit near unity $M_{\mathrm{r}} / M_{\mathrm{s}}$ ratios, $^{10}$ as discussed in Section 3.1. Second, the MBE growth of BaM thin films has the potential to achieve tight stoichiometric control and near-perfect crystal structures needed for the realization of extremely low losses. ${ }^{14}$ Future work on the use of MBE thin films as seed layers for the fabrication of high-quality thick films is also of great interest.

\subsection{Fabrication of BaM thick films on semiconductor substrates}

For certain applications, high-power handling or strong coupling require the use of BaM thick films, with a desired thickness range of several hundreds of microns. In 2008, Chen et al. demonstrated the feasibility of the fabrication of polycrystalline BaM thick films on $\mathrm{Si}$ wafers by the screen printing method. ${ }^{16,17}$ The fabrication process involved five main steps, (1) deposition of an amorphous $\mathrm{Al}_{2} \mathrm{O}_{3}$ buffer layer on a $\mathrm{Si}$ (001) substrate by magnetron sputtering, (2) spreading of a BaM/binder paste over a stencil onto the substrate, (3) drying of the "wet" film on a hot plate $\left(300-400{ }^{\circ} \mathrm{C}\right)$, (4) sintering at $1100{ }^{\circ} \mathrm{C}$ in air for 2 hours, and (5) annealing at $1150{ }^{\circ} \mathrm{C}$ in air for 10 minutes. The $\mathrm{Al}_{2} \mathrm{O}_{3}$ layer was about $1 \mu \mathrm{m}$ thick and served as a barrier to reduce the diffusion of $\mathrm{Si}$ into the BaM film during the sintering process. The printing paste consisted of 70-75 $\mathrm{wt} \%$ BaM particles and $25-30 \mathrm{wt} \%$ binder, and the $\mathrm{BaM}$ particles had an average size of $0.6 \mu \mathrm{m}$. The sintering at $1100{ }^{\circ} \mathrm{C}$ facilitated the growth of BaM grains and the formation of a continuous film. The annealing at $1150{ }^{\circ} \mathrm{C}$ allowed for a moderate level of diffusion of aluminum ions into the BaM lattice near the interface. This moderate diffusion enhanced the adhesion of the film to the substrate.

The polycrystalline BaM film wafers with a thickness range of $50-200 \mu \mathrm{m}$ and a diameter of 1 inch were fabricated. ${ }^{16,17}$ Representative hysteresis loops are shown in Fig. 13. ${ }^{17}$ The dashed and solid loops show the data obtained at $5 \mathrm{~K}$ and $300 \mathrm{~K}$, respectively. The data indicate a $4 \pi M_{s}$ value of $2090 \mathrm{G}$ at $5 \mathrm{~K}$ and a value of $1477 \mathrm{G}$ at $300 \mathrm{~K}$. These values are smaller than

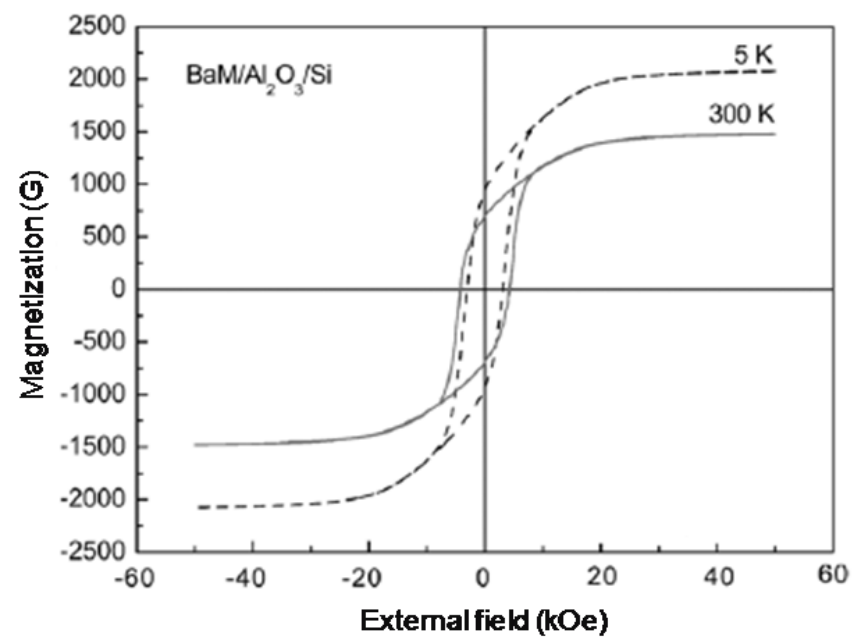

Fig. 13. Hysteresis loops for a BaM thick film grown on a Si substrate by screen printing. The dashed and solid loops show the data measured at $5 \mathrm{~K}$ and $300 \mathrm{~K}$, respectively. (After Ref. [17]) 
that for single-crystal BaM bulks. The main reason for this lies on the relatively low density of the film, which is intrinsic to the screen printing technique. The data also indicate a $M_{\mathrm{r}} / M_{\mathrm{s}}$ ratio of about 0.44 at $5 \mathrm{~K}$, which is close the expected value of 0.5 for randomly oriented polycrystalline samples.

Figure 14 shows the FMR linewidth vs. frequency response of a BaM thick film. ${ }^{16}$ One sees nearly linear behavior. All the linewidth values are larger than 1 kOe. Possible reasons for such large values include the following. (1) The magnetic fields used in FMR measurements were less than $20 \mathrm{kOe}$. As a result, the magnetization in the BaM film was not completely saturated. (2) The grains in the film were randomly oriented. (3) The porosity in the film was relatively high. Future work on the optimization of the screen printing process for BaM thick films with much narrower FMR linewidths is of significant interest.

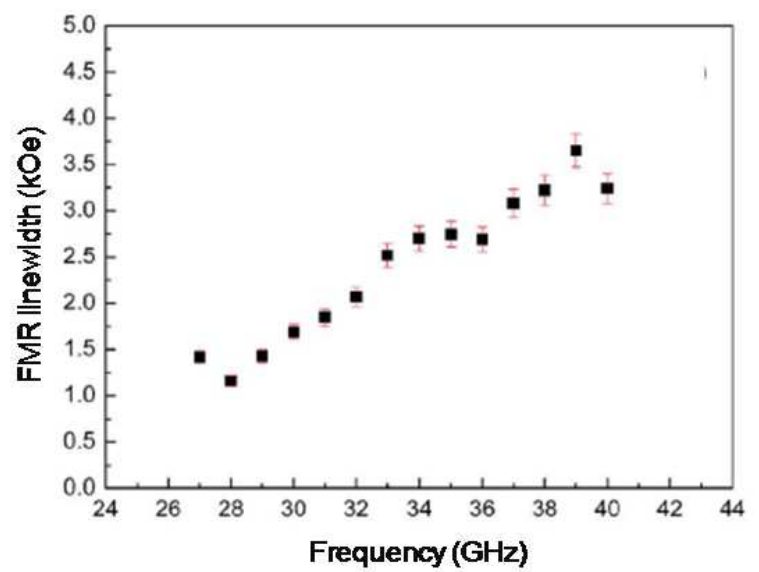

Fig. 14. FMR linewidth as a function of frequency for a BaM thick film fabricated on a Si substrate by screen printing. (After Ref. [16])

\section{BaM thin film-based millimeter wave devices}

Great progress has been made in recent years in the development of BaM-based planar mmwave devices.10,18-24 Such devices include (1) notch filters made of BaM thin films and microstrip geometry, (2) notch filters made of BaM slabs and strip-line geometry, (3) selfbiased notch filters using high- $M_{\mathrm{r}} \mathrm{BaM}$ thin films and coplanar waveguide (CPW) geometry, (4) notch filters based on the excitation of confined magnetostatic waves in narrow BaM strips and $\mathrm{CPW}$ configurations, and (5) phase shifters using BaM thin films and CPW geometry. This section describes the device configurations and responses of (3), (4), and (5). One can refer to Refs. [22] and [23] for details on (1) and (2).

\subsection{Self-biased millimeter wave notch filters}

Song et al. demonstrated a self-biased BaM notch filter in 2010.10 The device consisted of a high- $M_{\mathrm{r}}$ BaM film element positioned on the top of a CPW structure. The alternating magnetic field produced by the CPW signal line is spatially non-uniform. This non-uniform field excites magnetostatic waves (MSW) in the BaM film. ${ }^{1,3}$ Such waves propagate along the 
direction transversal to the CPW signal line and decay during the propagation. The net effect is a band-stop response of the device in a certain frequency range which corresponds to the bandwidth of the MSW excitation.

Figure 15 shows the structure and responses of such a notch filter. Figure 15(a) shows the device structure. Figure 15(b) shows a transmission profile of the device for zero external fields. Figure 15(c) shows transmission profiles measured at different external fields (Oe), as indicated. The BaM film element was $4.3 \mathrm{~mm}$ by $2.3 \mathrm{~mm}$. Its properties are discussed in Section 3.1. The longer side of the film element, which was also the easy axis direction, was along the CPW signal line. The width of the CPW signal line was $50 \mu \mathrm{m}$. For the data in Fig. 15(c), the fields were applied along the CPW signal line.
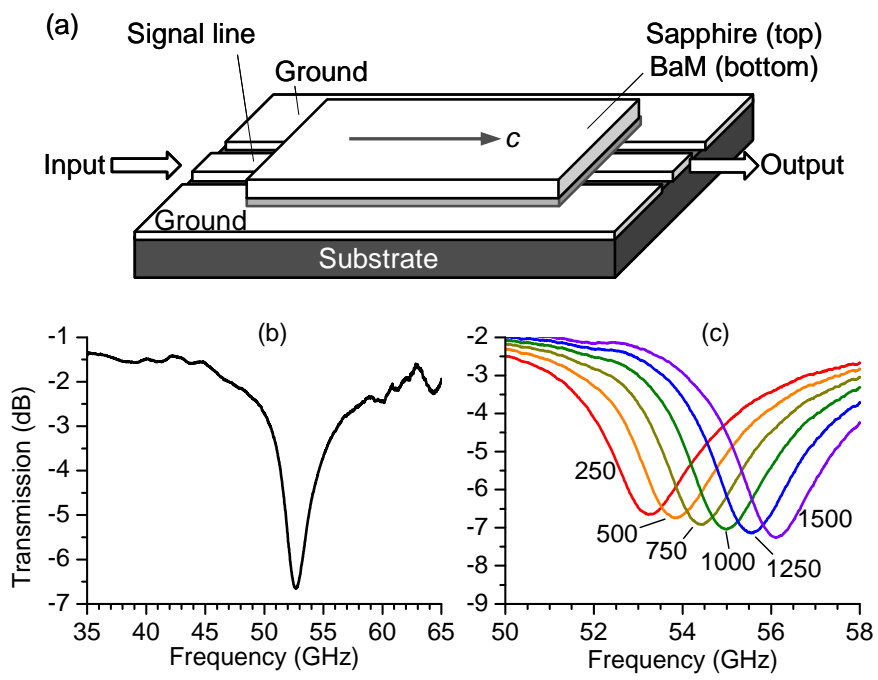

Fig. 15. (a) Diagram of a BaM thin film-based mm-wave notch filter. (b) Transmission profile of the filter for zero fields. (c) Transmission profiles of the filter for nonzero fields (Oe), as indicated. (After Ref. [10])

The data in Fig. 15(b) show a band-stop response, with a maximum absorption of $6.7 \mathrm{~dB}$ and an insertion loss less than $2 \mathrm{~dB}$. The center frequency is $52.69 \mathrm{GHz}$, which is slightly higher than the zero field FMR frequency of $52.50 \mathrm{GHz}$. This difference results from the fact that the frequency of the magnetostatic waves transverse to the field is above the FMR frequency. ${ }^{1,3}$ The data also show a $3 \mathrm{~dB}$ linewidth of about $2.52 \mathrm{GHz}$. This value is much larger than the frequency-swept FMR linewidth, $\Delta f_{\mathrm{FMR}}$, of the film, about $1.46 \mathrm{GHz}$; this indicates that the filtering response is not due to the FMR effect. Note that the linewidth $\Delta f_{\text {FMR }}$ was obtained by

$$
\Delta f_{\mathrm{FMR}}=\left(\left.\frac{\partial f_{\mathrm{FMR}}}{\partial H}\right|_{H=0}\right) \sqrt{3} \Delta H_{\mathbf{p p}}
$$

with $\Delta H_{\mathrm{pp}}=300$ Oe. These responses resulted from the self-biased operation of the device. The data in Fig. 15(c) indicate that the filter can be tuned for higher frequency operations with relatively low fields. Note that, for a YIG-based notch filter, an operation at $53 \mathrm{GHz}$ 
requires an external field of $18 \mathrm{kOe}$, which is significantly higher than the fields indicated in Fig. 15(c). It should also be noted that the insertion loss at the shoulders of the absorption dip results mainly from the ferromagnetic resonance. This is demonstrated by the decrease of the insertion loss at $52 \mathrm{GHz}$ with the increase of the field shown in Fig. 15(c).

\subsection{Notch filters based on excitation of confined magnetostatic waves}

The notch filter discussed in Section 4.1 showed a maximum absorption of only $6.7 \mathrm{~dB}$. In terms of practical applications, filters with much larger maximum absorption are desirable. In 2011, Lu et al. demonstrated that one could significantly increase the absorption level through the replacement of the wide BaM film element with a narrow BaM film strip. ${ }^{24}$ This significant increase in absorption results from the excitation of confined MSW modes in the BaM strip, which is explained in detail below.

Figure 16 shows the configuration of such a notch filter. The BaM film strip has its substrate facing up and its length along the CPW signal line. The easy axis of the BaM strip is along the strip length. The CPW-produced non-uniform magnetic field excites MSW modes in the BaM strip. These waves propagate along the strip width direction and are confined by the edges of the strip. The net effect is that the modes that satisfy the phase constraint $2 k w=n 2 \pi$ are relatively strong, while other modes are weak. In the phase condition, $k$ is the wavenumber, $w$ is the width of the BaM strip, and $n$ is an odd integer. The waveforms for the first three modes are shown schematically in Fig. 16. The excitation of these confined modes results in reduced output power and a band-stop filtering response which is discussed below.

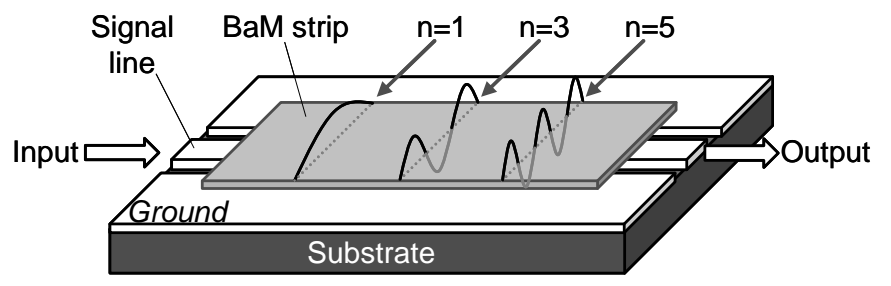

Fig. 16. Diagram of a confined magnetostatic wave-based BaM notch filter. (After Ref. [24])

There are two important points to be noted. (1) The even modes $(n=2,4,6 \ldots)$ cannot be excited. This is because the in-plane components of the CPW signal line-produced alternating magnetic field is symmetric across the signal line. (2) Only the $n=1$ mode will be strongly excited and other modes will be very weak. This is due to the fact that the MSW excitation efficiency decreases significantly with the wavenumber $k$ for the geometry considered here.

For the data described below, the coplanar waveguide has a $50 \mu \mathrm{m}$-wide signal line, a signal line-to-ground separation of $25 \mu \mathrm{m}$, and a nominal impedance of $50 \Omega$. A wide BaM film, which was the same as the films discussed in Sections 3.1 and 4.1, was cut into a narrow strip with a length of $4.30 \mathrm{~mm}$. The width of the BaM strip was reduced to different values through polishing and ranged from $1.30 \mathrm{~mm}$ to $0.24 \mathrm{~mm}$. The $\Delta H_{\mathrm{pp}}$ values for the $1.30 \mathrm{~mm}$ strip and the $0.24 \mathrm{~mm}$ strip were $306 \mathrm{Oe}$ and $307 \mathrm{Oe}$, respectively, at $56 \mathrm{GHz}$. The closeness of these two values indicates a good uniformity of the initial wide BaM film. With Eq. (3) 
and the parameters cited in Section 3.1, one can convert these $\Delta H_{\mathrm{pp}}$ values to a $\Delta f_{\mathrm{FMR}}$ value of about $1.49 \mathrm{GHz}$.

Figure 17 shows representative data on the self-biased operation of the device. Figure 17(a) gives the transmission profiles for filters with BaM strips of different widths, as indicated. Figures 17(b) and 17(c) give the maximum absorption and bandwidth data, respectively, for the filtering responses shown in Fig. 17(a). The bandwidth was taken at the transmission level $3 \mathrm{~dB}$ higher than the minimum transmission. Three important results are evident in Fig. 17. (1) The devices all show a self-biased band-stop response at about $52.7 \mathrm{GHz}$. (2) With a reduction in the $\mathrm{BaM}$ width, the maximum absorption increases substantially while the bandwidth decreases significantly. (3) In spite of the significant changes in absorption and bandwidth, there is only a slight increase in the device insertion loss. For all the measurements, the insertion loss is less than $2 \mathrm{~dB}$ on the low-frequency side and less than 4 $\mathrm{dB}$ on the high-frequency side.

The fact that a reduction in the BaM strip width leads to an increase in absorption and a decrease in bandwidth can be explained as follows. In a BaM strip which is relatively wide, the magnetostatic wave decays during its propagation along the width of the strip, and the MSW confinement across the strip width is weak. As a result, broadband magnetostatic waves are excited, and the filter shows a broad bandwidth and a small maximum absorption. In contrast, in a very narrow BaM strip, the effect of the MSW decay is insignificant and the MSW propagation is confined by the strip edges. This geometry confinement yields a phase constraint on the magnetostatic waves. As a result, narrowband magnetostatic waves with $k \approx \pi / w$ are excited, and the filter shows a much narrower bandwidth and a much larger absorption.
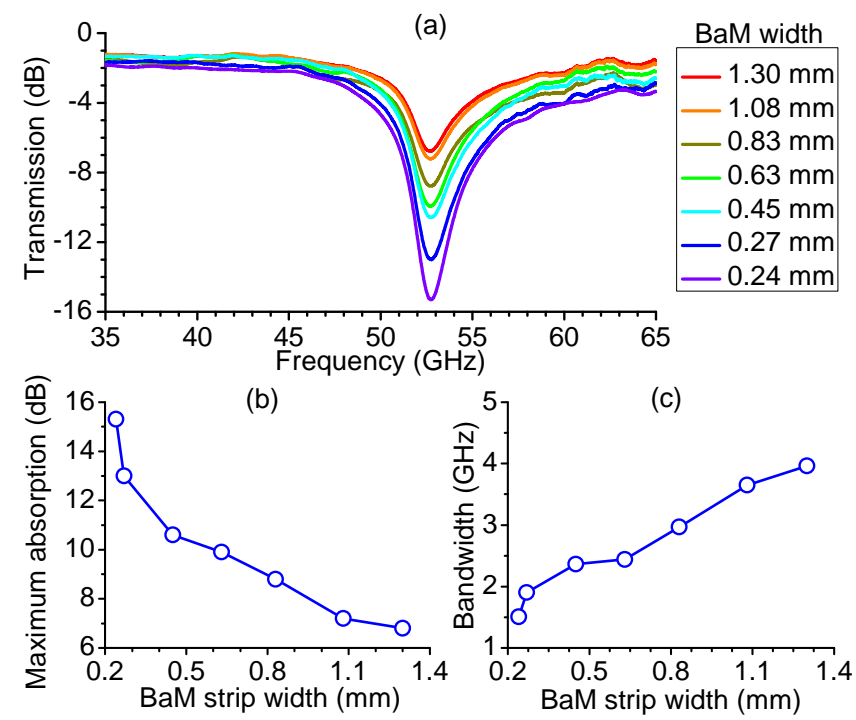

Fig. 17. (a) Transmission profiles for filters with BaM film strips of different widths. (b) Filter maximum absorption vs. BaM strip width. (c) Filter bandwidth vs. BaM strip width. (After Ref. [24]) 
The above explanation yields three expectations as follows. (1) As broadband magnetostatic waves are excited in wide BaM strips, the bandwidth of the filter using a wide BaM strip should be larger than the FMR linewidth $\Delta f_{\text {FMR }}$ of the BaM strip. (2) The field/film configuration considered here supports the propagation of surface MSW modes, for which the frequency $f$ is higher than $f_{\mathrm{FMR}}$ and increases with the wavenumber $k$. As a result, when one shifts from a regime where broadband magnetostatic waves are excited and the maximum absorption is at $f_{\mathrm{FMR}}$ to a regime where only the confined modes are excited and the center frequency is at $f=f_{k=\pi / w}$, one should see a shift of the maximum absorption frequency to a higher value. (3) One should also expect that, with a reduction in BaM strip width, the absorption profile is narrowed from the high frequency side, not the low frequency side, as the frequencies of the broadband MSW excitations are all above $f_{\mathrm{FMR}}{ }^{3}$

These expectations are all confirmed by the data in Fig. 17. (1) The bandwidths of the filters with wide strips are all larger than $\Delta f_{\mathrm{FMR}}$, while that of the filter with the $0.24 \mathrm{~mm}$ strip is just slightly larger than $\Delta f_{\text {FMR. }}$ (2) When the width was reduced from $1.30 \mathrm{~mm}$ to $0.24 \mathrm{~mm}$, the maximum absorption frequency increased from $52.72 \mathrm{GHz}$ to $52.74 \mathrm{GHz}$. The net increase agrees well with the theoretical value, which is evaluated as $f_{k=\pi / w}-f_{\mathrm{FMR}}=19 \mathrm{MHz}$. (3) With a reduction in strip width, the $3 \mathrm{~dB}$ bandwidth of the absorption profile is reduced on the high frequency side.

In addition to the self-biased operation described above, the filter is also tunable with low fields. Figure 18 demonstrates such low-field tuning. Figure 18(a) shows the transmission profiles for different external fields for a device with the $0.24 \mathrm{~mm}$-wide strip. Figures $18(\mathrm{~b})$ and 18(c) show the maximum absorption frequency and bandwidth, respectively, as a function of field for the filtering response. As for the data in Fig. 17(c), the bandwidth was taken at the transmission level $3 \mathrm{~dB}$ higher than the minimum transmission. For all the measurements, the fields were applied along the BaM strip. The curve in Fig. 18(b) shows MSW frequencies calculated as ${ }^{3}$

$$
f(H)=|\gamma|\left[\left(H+H_{a}\right)\left(H+H_{a}+4 \pi M_{s}\right)+\frac{1}{4}\left(4 \pi M_{s}\right)^{2}\left(1-e^{-2 \pi d / w}\right)\right]^{\frac{1}{2}}
$$

where $d$ is the film thickness. The calculations used the parameters cited above and those in Section 3.1. The only exception is that a value of $16.94 \mathrm{kOe}$ was used for $H_{\mathrm{a}}$.

The data in Fig. 18 show four things. (1) The filter is tunable with low fields. (2) The operation frequency increases almost linearly with field and matches almost perfectly with the theoretical value. (3) The bandwidth decreases slightly, with an overall change of only $7 \%$ in the entire field range. (4) The insertion loss remains almost constant. All of these results are critical for practical applications. The data in Fig. 18(a) also show a slight increase in maximum absorption with field. The fact that the absorption is lower and the bandwidth is larger at low fields may be a result of low-field loss effects. ${ }^{53}$

\subsection{Millimeter wave phase shifters}

The devices in both Sections 4.1 and 4.2 made use of CPW geometry. With similar geometry, it is also possible to make planar BaM phase shifters. This possibility was demonstrated by Wang et al. in 2010.21 The phase shifter was made of the same structure as 

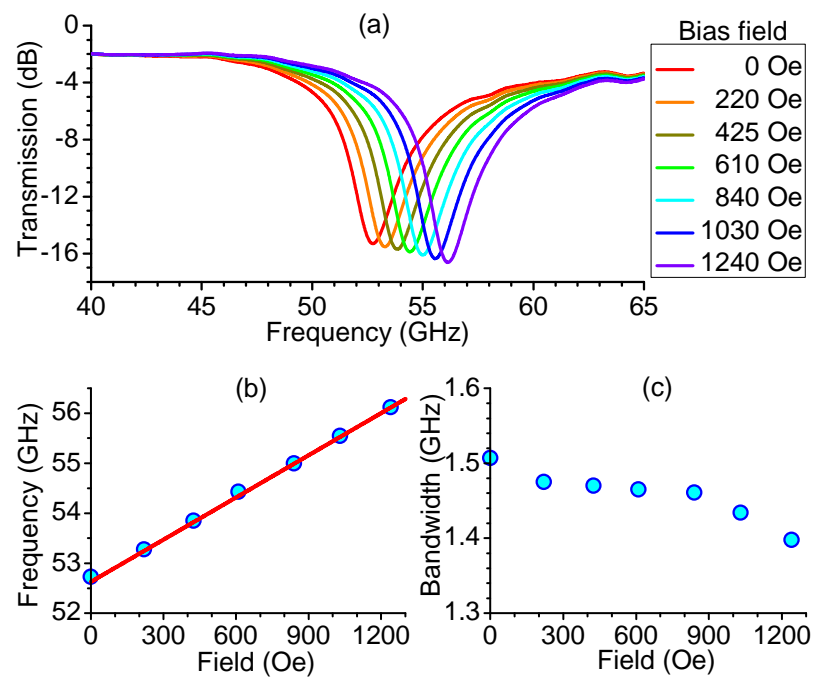

Fig. 18. (a) Transmission profiles measured at different fields, as indicated, for a filter with a $0.24 \mathrm{~mm}$-wide BaM strip. (b) Filter center frequency as a function of field. (c) Filter bandwidth as a function of field. The curve in (b) shows theoretical frequencies. (After Ref. [24])

that shown in Fig. 15(a). The coplanar waveguide had a length of $5.0 \mathrm{~mm}$ and an impedance of $50 \Omega$. The signal line had a width of $100 \mu \mathrm{m}$, and the separation between the signal line and grounds was $50 \mu \mathrm{m}$. The BaM film element was $4 \mathrm{~mm}$ long, $2 \mathrm{~mm}$ wide, and $5 \mu \mathrm{m}$ thick. It was positioned on the top of the coplanar waveguide with its substrate side facing up and its short edge parallel to the signal line. The film was grown on a $c$-plane sapphire substrate through PLD techniques. ${ }^{7}$ It showed out-of-plane $c$-axis orientation, an effective out-of-plane anisotropy field of $16.5 \mathrm{kOe}$, a saturation induction of $4300 \mathrm{G}$, and a $60 \mathrm{GHz}$ peak-to-peak FMR linewidth of 340 Oe. For the measurements of the phase shifter, an external field was applied perpendicular to the film plane to tune the phase of the signal.

Figure 19 shows the responses of the device. ${ }^{21}$ Figures 19(a) and 19(b) present the amplitude and phase, respectively, of the transmission parameter $S_{21}$ for four different fields. Figures 19(c) and 19(d) give the central frequency and $3 \mathrm{~dB}$ linewidth of the dips in the transmission profiles, four of which are shown in Fig. 19(a). The line in Fig. 19(c) shows the theoretical FMR frequencies of the BaM film calculated with Eq. (1), the parameters given above, and a small field correction of $+0.14 \mathrm{kOe}$. It should be noted that previous theoretical calculations for microstrip geometry had shown that the position of the dip in transmission did not necessarily occur at the same frequency as the maximum absorption in a FMR experiment. ${ }^{20}$ The field correction found here is consistent, in magnitude and sign, with the shift found in Ref. [20].

The data in Fig. 19 show four results. (1) As shown in Fig. 19(a), the transmission of the device shows a clear dip response, which is tunable with the field. (2) In the dip regime, the phase changes notably with the field. (3) There is a good agreement between the dip central frequencies and theoretical FMR frequencies. This match indicates that the transmission dip and phase change responses originate from the FMR effect in the BaM film. (4) The linewidth is one order of magnitude higher than and shows a frequency dependence opposite to that reported in Ref. [7]. 

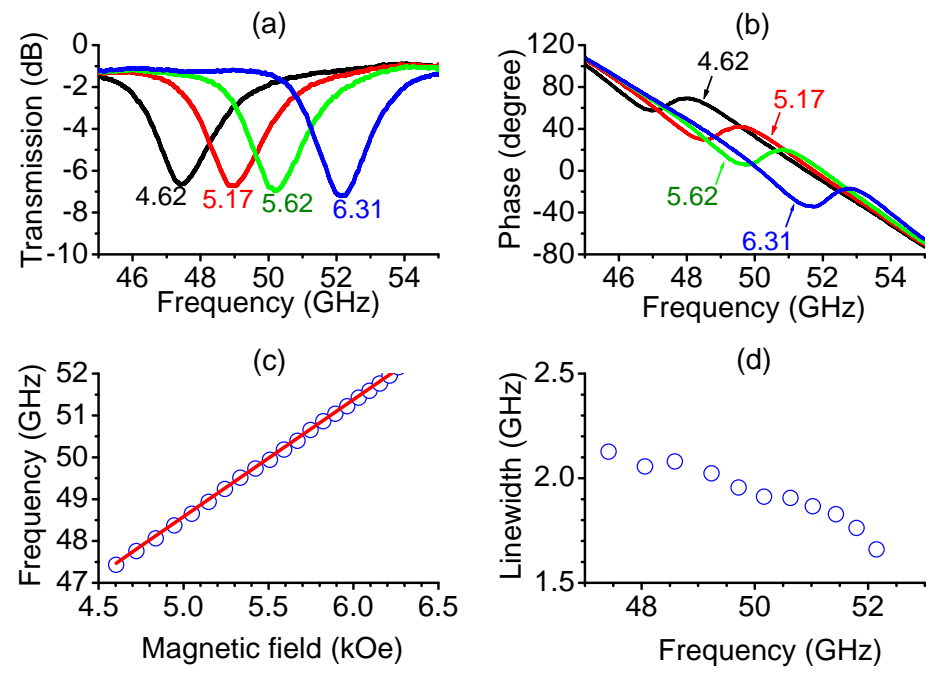

Fig. 19. Responses of a BaM-based mm-wave phase shifter. (a) and (b) show the transmission and phase profiles, respectively, which were measured for four different fields (kOe), as indicated. (c) and (d) show the frequency and $3 \mathrm{~dB}$ linewidth for the dips in the transmission profiles shown representatively in (a). (After Ref. [21])

Figure 20 shows representative data on the phase shift properties. Figures 20(a) and 20(b) show the phase shift and insertion loss, respectively, as a function of field measured at $50 \mathrm{GHz}$. The phase shift is the phase of the device output signal relative to that measured at $H=5.35$ kOe. Figures 20(c) and 20(d) show the data for $54 \mathrm{GHz}$. The phase shift in Fig. 20(c) is the phase relative to that measured at $4.60 \mathrm{kOe}$. For the field ranges in Fig. 20, the $50 \mathrm{GHz}$ point is within the on-resonance regime, while the $54 \mathrm{GHz}$ point is in the below-resonance regime.

The data in Figs. 20(a) and 20(b) show a negative phase shift which decreases almost linearly with $H$ and an insertion loss which is almost constant over the entire field range. The phase shift decreased from 0 to $-30^{\circ}$ as the field was increased from $5.35 \mathrm{kOe}$ to $5.70 \mathrm{kOe}$. This corresponds to a phase tuning rate of $43^{\circ} /(\mathrm{mm} \cdot \mathrm{kOe})$. The insertion loss changed in a narrow range of $6.3 \pm 0.5 \mathrm{~dB}$, and this corresponds to a loss rate of about $3.1 \mathrm{~dB} / \mathrm{mm}$. The responses at $54 \mathrm{GHz}$ are significantly different. Specifically, both the phase shift and insertion loss are smaller than those for $50 \mathrm{GHz}$. The linear phase tuning range is $11^{\circ}$. The phase tuning rate is $3.2^{\circ} /(\mathrm{mm} \cdot \mathrm{kOe})$. The insertion loss is only $0.7 \mathrm{~dB} / \mathrm{mm}$.

The above results clearly demonstrate the feasibility of the use of FMR effects in BaM thin films to develop planar phase shifters for mm-wave signal processing. Three points should be mentioned. (1) It is the built-in high anisotropy field that facilitates the operation of the phase shifter at $50 \mathrm{GHz}$ for relatively low bias fields. (2) Both the linear tuning of the phase and the flatness of the loss curves are critical for practical applications. The maximal phase shift ranges are much wider than those shown in Fig. 20. In these wider ranges, however, the phase tuning is nonlinear and the loss is not constant. (3) Strictly speaking, the modes in the BaM film were MSW modes, as those discussed in Section 4.1, rather than the uniform 
FMR modes. In fact, as the CPW signal line was relatively wide and the BaM film had a relatively broad FMR linewidth, one can view those MSW modes as quasi-FMR modes.
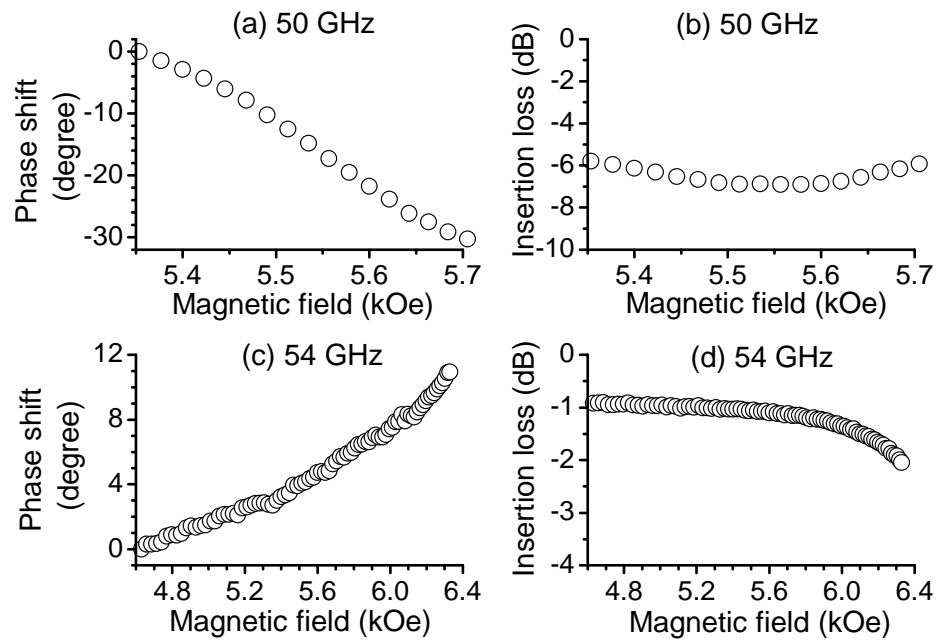

Fig. 20. Phase shift and insertion loss of a BaM-based phase shifter as a function of bias field. The data in (a) and (b) were measured at a fixed frequency of $50 \mathrm{GHz}$. The data in (c) and (d) were measured at $54 \mathrm{GHz}$. (After Ref. [21])

\subsection{Theoretical analysis of millimeter wave phase shifters}

Camley et al. carried out theoretical studies on the feasibility of BaM thin film-based mmwave devices. ${ }^{20,21,22}$ Figure 21 shows representative theoretical results obtained for a phase shifter that had the same structure and parameters as the one described in Section 4.3.21 Figures 21(a) and 21(b) give the transmission and phase profiles, respectively, of the device for different fields. Figures 21(c) and 21(d) give the phase shift as a function of field for different frequencies. For a better comparison with experimental data, these theoretical data are shown in the same format as those shown in Figs. 19 and 20. The calculations were carried out with an effective medium theory ${ }^{54}$ and the parameters cited in Section 4.3. The permeability for the BaM film was given in Ref. [20]. The FMR linewidth of the BaM film was taken to be $\Delta H_{\mathrm{FMR}}=\Delta H_{0}+2 \alpha f /|\gamma|$, where $\Delta H_{0}$ was the frequency independent linewidth, chosen as $400 \mathrm{Oe}, \alpha$ was the damping constant, chosen as 0.0042 , and $f$ was the frequency. The dielectric constant for the BaM film and the substrates was chosen as 10 . The effective medium was composed of a $5 \mu \mathrm{m}$-thick BaM film, a $150 \mu \mathrm{m}$-thick sapphire layer, and an air gap of $5 \mu \mathrm{m}$ between the BaM film and coplanar waveguide. The air gap was considered because the BaM film was positioned, not deposited, on the coplanar waveguide.

The data in Figs. 21(a) and 21(b) indicate the following results. (1) The device shows a fieldtunable resonance response. (2) The transmission dip is slightly asymmetrical at low fields. (3) The minimum transmission is about $-7 \mathrm{~dB}$ and decreases slightly with frequency. (4) The width of the transmission dip decreases slightly with frequency. (5) The phase increases about $33^{\circ}$ during the resonance. All of these results agree very well with the experimental 


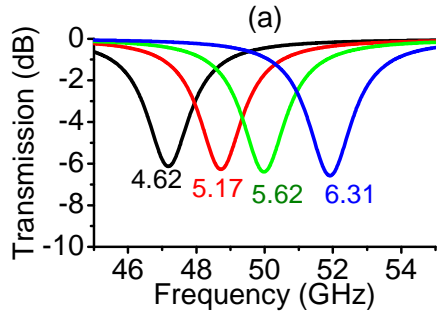

(c)

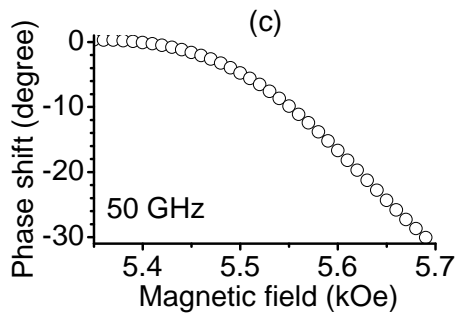

(b)

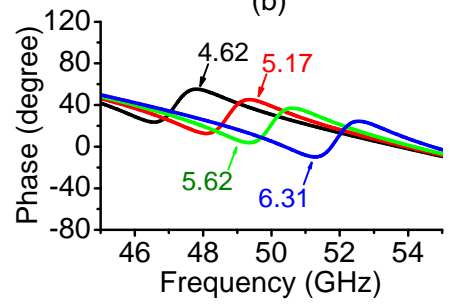

(d)

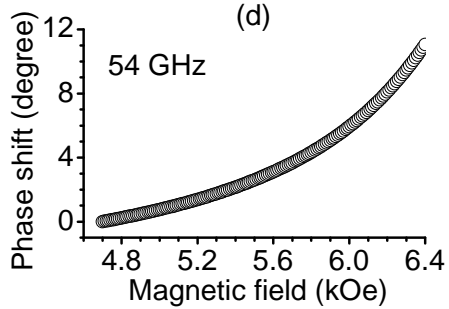

Fig. 21. Simulation of a BaM-based mm-wave phase shifter. (a) and (b) show the device transmission and phase profiles, respectively, for four different fields (kOe). (c) and (d) show the phase shift as a function of field for $50 \mathrm{GHz}$ and $54 \mathrm{GHz}$, respectively. (After Ref. [21])

results shown in Fig. 19. However, both the off-resonance insertion loss and the entire phase change are smaller than experimental values. This is because the calculations considered only the short active portion of the device - the portion with the BaM film, not the $5 \mathrm{~mm}$ long coplanar waveguide. The data in Figs. 21(c) and 21(d) show the phase shift ranges and tuning rates of the device that perfectly match the experimental values shown in Fig. 20.

One can see that the theoretical results agree with the measurements of the actual phase shifter. In addition to the analysis on the phase shifter, Camley et al. also conducted theoretical analysis on microstrip-type BaM notch filters. ${ }^{20,22}$ The results, which are not discussed here, were very close to those measured experimentally. ${ }^{22}$

\section{Outlook}

Recent years have witnessed significant progress in the development of low-loss, self-biased $\mathrm{BaM}$ thin films, the deposition of high-quality $\mathrm{BaM}$ thin films on metallic and semiconductor substrates, the fabrication of BaM thick films on semiconductor substrates, and the development of BaM thin film-based planar mm-wave devices. Although not reviewed in this chapter, significant advances have also been made in the development of $\mathrm{BaM} /$ ferroelectric and $\mathrm{BaM} /$ piezoelectric heterostructures and the electrical tuning of magnetic responses therein, as well as in the study of multiferroic effects in BaM materials. These advances have far-reaching implications for the future development of BaM filmbased devices for mm-wave signal processing. It is believed that BaM film-based devices will greatly impact the advancement of the mm-wave technology, just as YIG devices had impacted the microwave technology in the past decades.

In lieu of those significant advances, there exist many future research works that are of great interest and importance in terms of practical applications. These include, but not limited to, the following topics. Some of them have already been mentioned in previous sections. 
1. Development of BaM thin films that have low loss and high remanent magnetization, as the films discussed in Section 3.1, but with either lower or higher anisotropy fields. This work is important for the development of self-biased devices for applications in different frequency regimes. It can be realized through the doping of scandium or aluminum into BaM films. 51,52

2. Fabrication of high-quality BaM thin films in "conventional" metallic substrates. This effort is critical for the development of mm-wave devices where a ground plane is requisite.

3. Deposition of high-quality BaM films on semiconductor substrates through the use of MBE BaM seed layers.

4. Optimization of screen printing processes for the fabrication of high-quality, thick BaM films on semiconductor substrates.

5. Development of BaM thin film-based, planar, non-reciprocal devices, such as isolators and circulators. Such devices are critically needed by mm-wave signal processing and can be realized through the use of coupled-line geometry. $.5,56$

6. Development of electrically tunable $\mathrm{BaM}$ mm-wave devices by the use of $\mathrm{BaM} /$ ferroelectric or $\mathrm{BaM} /$ piezoelectric heterostructures. Electrically tunable devices can have a number of advantages over devices that are tuned magnetically. These advantages include fast tuning, negligible power consumption, small size, and light weight.

\section{Acknowledgments}

The author wants to thank his colleagues Dr. Robert Camley, Mr. Lei Lu, Dr. Young-Yeal Song, Mr. Yiyan Sun, and Mr. Zihui Wang for meaningful scientific collaborations in the field of BaM thin films and devices. The author would like to particularly thank Dr. YoungYeal Song for extensive useful discussions. The author also thanks Dr. Yan Nie, Mr. Michael Kabatek, and Mr. James Hitchman for proofreading this chapter. The author apologizes to those who contributed to this research field and whose results are not included in this chapter. This omission results from the author's inability to include all results into this chapter which has a length constraint. Support from the U.S. National Science Foundation, the U.S. Army Research Office, the U.S. National Institute of Standards and Technology, and Colorado State University is gratefully acknowledged.

\section{References}

[1] P. Kabos and V. S. Stalmachov, Magnetostatic Waves and Their Applications (Chapman \& Hall, London, 1994).

[2] J. D. Adam, L. E. Davis, G. F. Dionne, E. F. Schloemann, and S. N. Stitzer, IEEE Trans. Microwave Theory Tech. 50, 721 (2002).

[3] D. D. Stancil and A. Prabhakar, Spin Waves: Theory and Applications (Springer, New York, 2009).

[4] F. Sammoura, Micromachined Plastic Millimeter-Wave Radar Components (VDM Verlag, Saarbrücken, 2008).

[5] D. Liu, U. Pfeiffer, J. Grzyb, and B. Gaucher, Advanced Millimeter-Wave Technologies: Antennas, Packaging and Circuits (Wiley, Chichester, 2009).

[6] U. Soerqel, Radar Remote Sensing of Urban Areas (Springer, New York, 2010). 
[7] Y. Y. Song, S. Kalarickal, and C. E. Patton, J. Appl. Phys. 94, 5103 (2003).

[8] S. D. Yoon, C. Vittoria, and S. A. Oliver, J. Appl. Phys. 93, 4023 (2003).

[9] S. D. Yoon, C. Vittoria, and S. A. Oliver, J. Magn. Magn. Mater. 265, 130 (2003).

[10] Y. Y. Song, Y, Sun, L. Lu, J. Bevivino, and M. Wu, Appl. Phys. Lett. 97, 173502 (2010).

[11] Z. Chen, A. Yang, S. D. Yoon, K. Ziemer, C. Vittoria, and V. G. Harris, J. Magn. Magn. Mater. 301, 166 (2006).

[12] Z. Chen, A. Yang, Z. Cai, S. D. Yoon, K. Ziemer, C. Vittoria, and V. G. Harris, IEEE Trans. Magn. 42, 2855 (2006).

[13] Z. Chen, A. Yang, A. Gieler, V. G. Harris, C. Vittoria, P. R. Ohodnicki, K. Y. Goh, M. E. Mchenry, Z. Cai, T. L. Goodrich, and K. S. Ziemer, Appl. Phys. Lett. 91, 182505 (2007).

[14] Z. Cai, T. L. Goodrich, B. Sun, Z. Chen, V. G. Harris, and K. S. Ziemer, J. Phys. D: Appl. Phys. 43, 095002 (2010).

[15] Y. Nie, I. Harward, K. Kalin, A. Beaubien, and Z. Celinski, J. Appl. Phys. 107, 073903 (2010).

[16] Y. Chen, I. C. Smith, A. L. Geiler, C. Vittoria, V. Zagorodnii, Z. Celinski, and V. G. Harris, IEEE Trans. Magn. 44, 4571 (2008).

[17] Y. Chen, I. C. Smith, A. L. Geiler, C. Vittoria, V. Zagorodnii, Z. Celinski, and V. G. Harris, J. Phys. D: Appl. Phys. 41, 095006 (2008).

[18] N. Zeina, H. How, and C. Vittoria, IEEE Trans. Magn. 28, 3219 (1992).

[19] S. Capraro, J. P. Chatelon, M. Le Berre, T. Rouiller, H. Joisten, D. Barbier, and J. J. Rousseau, Phys. Status Solidi C 1, 3373 (2004).

[20] T. J. Fal and R. E. Camley, J. Appl. Phys. 104, 023910 (2008).

[21] Z. Wang, Y. Y. Song, Y. Sun, J. Bevivino, M. Wu, V. Veerakumar, T. Fal, and R. Camley, Appl. Phys. Lett. 97, 072509 (2010).

[22] R. E. Camley, Z. Celinski, T. Fal, A. V. Glushchenko, A. J. Hutchison, Y. Khivintsev, B. Kuanr, I. R. Harward, V. Veerakumar, and V. V. Zagorodnii, J. Magn. Magn. Mater. 321, 2048 (2009).

[23] Y. Y. Song, C. L. Ordóñez-Romero, and M. Wu, Appl. Phys. Lett. 95, 142506 (2009).

[24] L. Lu, Y. Y. Song, J. Bevivino, and M. Wu, Appl. Phys. Lett. 98, 212505 (2011).

[25] J. Das, B. Kalinikos, A. R. Barman, and C. E. Patton, Appl. Phys. Lett. 91, 172516 (2007).

[26] A. B. Ustinov and G. Srinivasan, Appl. Phys. Lett. 93, 142503 (2008).

[27] Y. Y. Song, J. Das, P. Krivosik, N. Mo, and C. E. Patton, Appl. Phys. Lett. 94, 182502 (2009).

[28] J. Das, Y. Y. Song, and M. Wu, J. Appl. Phys. 108, 043911 (2010).

[29] Y. Y. Song, J. Das, P. Krivosik, H. K. Seo, and M. Wu, IEEE Magn. Lett. 1, 2500204 (2010).

[30] Y. Tokunaga, Y. Kaneko, D. Okuyama, S. Ishiwata, T. Arima, S. Wakimoto, K. Kakurai, Y. Taguchi, and Y. Tokura, Phys. Rev. Lett. 105, 257201 (2010).

[31] S. R. Shinde, R.Ramesh, S. E. Lofland, S. M. Bhagat, S. B. Ogale, R. P. Sharma, and T. Venkatesan, Appl. Phys. Lett. 72, 3443 (1998).

[32] S. D.Yoon and C.Vittoria, J. Appl. Phys. 93, 8597 (2003).

[33] S. D.Yoon and C.Vittoria, IEEE Trans. Magn. 39, 3163 (2003).

[34] S. D.Yoon and C.Vittoria, J. Appl. Phys. 96, 2131 (2004).

[35] Y. Y. Song, J. Das, Z. Wang, W. Tong, and C. E. Patton, Appl. Phys. Lett. 93, 172503 (2008). 
[36] M. S. Yuan, H. L. Glass, and L. R. Adkins, Appl. Phys. Lett. 53, 340 (1988).

[37] Z. Zhuang, M. Rao, R. M. White, D. E. Laughlin, and M. H. Kryder, J. Appl. Phys. 87, $6370(2000)$.

[38] S. Capraro, J. P. Chatelon, M. Le Berre, T. Rouiller, H. Joisten, D. Barbier, and J. J. Rousseau, Phys. Status Solidi C 1, 3373 (2004).

[39] N. N. Shams, M. Matsumoto, and A. Morisako, IEEE Trans. Magn. 40, 2955 (2004).

[40] N. N. Shams, X. Liu, M. Matsumoto, and A. Morisako, J. Appl. Phys. 97, 10K305 (2005).

[41] S. Pignard, J. P. Senateur, H. Vincent, J. Kreisel, and A. Abrutis, J. Phys. IV France 7, C1483 (1997).

[42] J. R. Hook and H. E. Hall, Solid State Physics (John Wiley \& Sons, New York, 1991)

[43] C. Kittel, Solid State Physics (John Wiley \& Sons, New York, 2005).

[44] J. Smit and H. P. J. Wijn, Ferrites (John Wiley \& Sons, New York, 1959).

[45] W. H. von Aulock, Handbooks of Microwave Ferrite Materials (Academic Press, New York, 1965).

[46] K. Okumura, T. Ishikura, M. Soda, T. Asaka, H. Nakamura, Y. Wakabayashi, and T. Kimura, Appl. Phys. Lett. 98, 212504 (2011).

[47] R. Karim, K. D. McKinstry, J. R. Truedson, and C. E. Patton, IEEE Trans. Magn. 28, 3225 (1992).

[48] M. A. Wittenauer, J. A. Nyenhuis, A. I. Schindler, H. Sato, F. J. Friedlaender, J. Truedson, R. Karim, and C. E. Patton, J. Cryst. Growth 130, 533 (1993).

[49] M. Sparks, Ferromagnetic-Relaxation Theory (McGraw Hill, New York, 1964).

[50] A. G. Gurevich and G. A. Melkov, Magnetization Oscillations and Waves (CRC-Press, Boca Raton, 1996).

[51] X. Zuo, P. Shi, S. A. Oliver, and C. Vittoria, J. Appl. Phys. 91, 7622 (2002).

[52] A. B. Ustinov, A. S. Tatarenko, G. Srinivasan, and A. M. Balbashov, J. Appl. Phys. 105, 023908 (2009).

[53] S. S. Kalarickal, N. Mo, P. Krivosik, and C. E. Patton, Phys. Rev. B 79, 094427 (2009).

[54] T. J. Fal, V. Veerakumar, Bijoy Kuanr, Y. V. Khivintsev, Z. Celinski, and R. E. Camley, J. Appl. Phys. 102, 063907 (2007).

[55] L. E. David and D. B. Sillars, IEEE Trans. Microw. Theory Tech. 34, 804 (1986).

[56] J. Mazur, M. Solecka, M. Mazur, R. Poltorak, and E. Sedek, IEE Proc. Microw. Antennas Propag. 152, 43 (2005). 


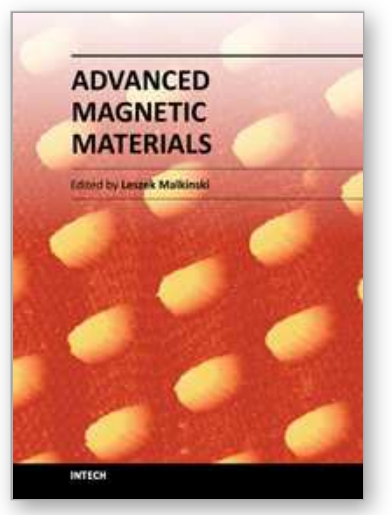

\author{
Advanced Magnetic Materials \\ Edited by Dr. Leszek Malkinski
}

ISBN 978-953-51-0637-1

Hard cover, 230 pages

Publisher InTech

Published online 24, May, 2012

Published in print edition May, 2012

This book reports on recent progress in emerging technologies, modern characterization methods, theory and applications of advanced magnetic materials. It covers broad spectrum of topics: technology and characterization of rapidly quenched nanowires for information technology; fabrication and properties of hexagonal ferrite films for microwave communication; surface reconstruction of magnetite for spintronics; synthesis of multiferroic composites for novel biomedical applications, optimization of electroplated inductors for microelectronic devices; theory of magnetism of Fe-Al alloys; and two advanced analytical approaches for modeling of magnetic materials using Everett integral and the inverse problem approach. This book is addressed to a diverse group of readers with general background in physics or materials science, but it can also benefit specialists in the field of magnetic materials.

\title{
How to reference
}

In order to correctly reference this scholarly work, feel free to copy and paste the following:

Mingzhong Wu (2012). M-Type Barium Hexagonal Ferrite Films, Advanced Magnetic Materials, Dr. Leszek Malkinski (Ed.), ISBN: 978-953-51-0637-1, InTech, Available from:

http://www.intechopen.com/books/advanced-magnetic-materials/m-type-barium-hexagonal-ferrite-films

\section{INTECH}

open science | open minds

\section{InTech Europe}

University Campus STeP Ri

Slavka Krautzeka 83/A

51000 Rijeka, Croatia

Phone: +385 (51) 770447

Fax: +385 (51) 686166

www.intechopen.com

\section{InTech China}

Unit 405, Office Block, Hotel Equatorial Shanghai

No.65, Yan An Road (West), Shanghai, 200040, China

中国上海市延安西路65号上海国际贵都大饭店办公楼 405 单元

Phone: +86-21-62489820

Fax: +86-21-62489821 
(C) 2012 The Author(s). Licensee IntechOpen. This is an open access article distributed under the terms of the Creative Commons Attribution 3.0 License, which permits unrestricted use, distribution, and reproduction in any medium, provided the original work is properly cited. 